\title{
Dualities near the horizon
}

\author{
Sergio Ferrara, ${ }^{a, b}$ Alessio Marrani, ${ }^{c}$ Emanuele Orazi $^{d}$ and Mario Trigiante ${ }^{e}$ \\ a Physics Department, Theory Unit, CERN, \\ CH 1211, Geneva 23, Switzerland \\ ${ }^{b}$ INFN - Laboratori Nazionali di Frascati, \\ Via Enrico Fermi 40, I-00044 Frascati, Italy \\ ${ }^{c}$ Instituut voor Theoretische Fysica, KU Leuven, \\ Celestijnenlaan 200D, B-3001 Leuven, Belgium \\ ${ }^{d}$ International Institute of Physics, Federal University of Rio Grande do Norte, \\ Av. Odilon Gomes de Lima 1722, Capim Macio, Natal-RN 59078-4 00, Brazil \\ ${ }^{e}$ Dipartimento di Fisica, Politecnico di Torino, and INFN — Sezione di Torino, \\ Corso Duca degli Abruzzi 24, I-10129 Torino, Italy \\ E-mail: sergio.ferrara@cern.ch, alessio.marrani@fys.kuleuven.be, \\ orazi.emanuele@gmail.com, mario.trigiante@polito.it
}

ABSTRACT: In 4-dimensional supergravity theories, covariant under symplectic electricmagnetic duality rotations, a significant role is played by the symplectic matrix $\mathcal{M}(\varphi)$, related to the coupling of scalars $\varphi$ to vector field-strengths. In particular, this matrix enters the twisted self-duality condition for 2-form field strengths in the symplectic formulation of generalized Maxwell equations in the presence of scalar fields.

In this investigation, we compute several properties of this matrix in relation to the attractor mechanism of extremal (asymptotically flat) black holes. At the attractor points with no flat directions (as in the $\mathcal{N}=2$ BPS case), this matrix enjoys a universal form in terms of the dyonic charge vector $\mathcal{Q}$ and the invariants of the corresponding symplectic representation $\mathbf{R}_{\mathcal{Q}}$ of the duality group $G$, whenever the scalar manifold is a symmetric space with $G$ simple and non-degenerate of type $\mathrm{E}_{7}$.

At attractors with flat directions, $\mathcal{M}$ still depends on flat directions, but not $\mathcal{M Q}$, defining the so-called Freudenthal dual of $\mathcal{Q}$ itself. This allows for a universal expression of the symplectic vector field strengths in terms of $\mathcal{Q}$, in the near-horizon Bertotti-Robinson black hole geometry.

KEYWORDS: Extended Supersymmetry, Supergravity Models

ArXiv EPRINT: 1305.2057 


\section{Contents}

1 Introduction 1

2 Symmetry properties of extremal black holes in extended supergravities 5

3 The $M$-matrix and $\mathcal{M}^{H}$ without flat directions $\quad 10$

3.1 The $I_{4}>0$ case and $\mathcal{M}^{H} \quad 11$

3.2 Generalizing the solutions $M_{ \pm}$to all $I_{4} \neq 0$ orbits 17

$\begin{array}{lll}3.3 & \text { Interpretation of } M_{ \pm} \text {in } \mathcal{N}=2 \text { theories } & 19\end{array}$

4 General discussion and summary of results 20

A The $K$-tensor $\quad 23$

B Computing the coefficients $A, B$ and $C \quad 25$

B.1 With the invariant tensor $S_{M Q}^{\alpha \beta} \ldots \quad 25$

$\begin{array}{lll}\text { B.2 } \ldots \text { and without } S_{M Q}^{\alpha \beta} & 27\end{array}$

$\begin{array}{lr}\text { C Signature of } M_{-} & 28\end{array}$

$\begin{array}{ll}\text { D Outer (anti-symplectic) automorphisms of } \mathfrak{g} & 28\end{array}$

\section{Introduction}

One of the most appealing properties of extended (ungauged) four-dimensional supergravities (i.e. locally supersymmetric models with no less than 8 supercharges) is their on-shell global symmetry which is conjectured to encode the known string/M-theory dualities [1]. The corresponding global symmetry group $G$, to be also dubbed $U$-duality, is the isometry group of the scalar manifold (i.e., global symmetry of the scalar field sigma-model), whose (non-linear) action on the scalar fields is combined with a linear symplectic action on the $n$ electric field strengths $F_{\mu \nu}^{\Lambda}, \Lambda=0, \ldots, n-1$, and their magnetic duals $G_{\Lambda \mid \mu \nu}[2]$ (electric-magnetic duality action of $G$ ). The latter action being defined by an embedding of $G$ in the symplectic group $\operatorname{Sp}(2 n, \mathbb{R})$, so that $F_{\mu \nu}^{\Lambda}$, together with $G_{\Lambda \mu \nu}$, transform under electric-magnetic duality in a symplectic representation $\mathbf{R}_{\mathcal{Q}}$ of $G$. This embedding, which determines the couplings of the vector fields to all the other fields in the action, is built-in the definition of a flat symplectic bundle over the scalar manifold, which is a common mathematical feature of $\mathcal{N} \geqslant 2$-extended supergravities [3-5].

Solutions to these theories naturally arrange themselves in orbits with respect to the action of $G$, and important physical properties are captured by $G$-invariant quantities. A notable example are the extremal, static, asymptotically-flat black holes in $D=4$, 
which have deserved considerable attention in the literature during the last 20 years or so, since they provide a valuable tool for studying string/M-theory dualities. These solutions are naturally coupled to scalar fields as a consequence of the non-minimal couplings of these to the vector fields in the supergravity action. Near the horizon, however, they exhibit an attractor mechanism [6-10]: the near-horizon geometry, which is described by an $A d S_{2} \times S^{2}$ Bertotti-Robinson space-time [11], is independent of the values of the scalar fields at radial infinity, and only depends on the quantized magnetic and electric charges $p^{\Lambda}, q_{\Lambda}$. In particular the horizon area $A_{H}$, which is related to the entropy $S$ of the solution through the Bekenstein- Hawking formula $[12,13]$, is expressed in terms of the quartic invariant $I_{4}(p, q)$ of the representation $\mathbf{R}_{\mathcal{Q}}$ of $G$, only depending on $p^{\Lambda}, q_{\Lambda}$ (we set $8 \pi G_{N}=$ $c=\hbar=1)$ :

$$
S=\frac{A_{H}}{4}=\pi \sqrt{\left|I_{4}(p, q)\right|} .
$$

This is a consequence of the fact that the horizon represents an asymptotically stable equilibrium point for the radial evolution of those scalar fields which are effectively coupled to the solution and thus affect its geometry. In other words, such scalars flow from radial infinity to the horizon toward values which only depend on the quantized charges (fixed values). The horizon fixed point is defined by extremizing an effective potential $V_{B H}(\varphi ; p, q)$ ( $\varphi$ generically denoting the scalar fields) [10]:

$$
V_{B H}(\varphi, \mathcal{Q}):=-\frac{1}{2} \mathcal{Q}^{T} \mathcal{M}(\varphi) \mathcal{Q}
$$

where $\mathcal{Q}=\left(p^{\Lambda}, q_{\Lambda}\right)$ is the vector quantized charges in the representation $\mathbf{R}_{\mathcal{Q}}$ of $G$. The value of this potential at the horizon defines its area, being equal to $\sqrt{\left|I_{4}(p, q)\right|}$. The scalar fields which are not fixed at the horizon are those which are not effectively coupled to the black hole charges, and they are flat directions of $V_{B H}$. They will be denoted by $\varphi_{\text {flat }}$. In the above formula, $\mathcal{M}(\varphi)$ is a $2 n \times 2 n$ symmetric, symplectic, negative-definite matrix-valued function of the scalar fields. In all extended supergravities it is defined by the flat symplectic bundle over the scalar manifold. In fact, it encodes all the information about the non-minimal couplings of the scalar to the vector fields in the action through the kinetic term of the latter and the generalized theta-term. Moreover it allows to define the so called Freudenthal duality [14], extensively studied in [15-17], which we shall be dealing with in the following.

An interesting question to be posed is what happens to the geometric structures associated with the scalar manifold, e.g. pertaining to its symplectic bundle, near the horizon. In the present investigation, we focus on the matrix $\mathcal{M}(\varphi)$, because of its relevance to the geometry of the supergravity model.

At the horizon $\mathcal{M}(\varphi)$ depends on $\mathcal{Q}$, through the fixed scalars, and on the flat directions:

$$
\left.\mathcal{M}(\varphi)\right|_{\text {horizon }}=\mathcal{M}^{H}\left(\mathcal{Q}, \varphi_{\text {flat }}\right) .
$$

The dependence on the flat directions drops out already when we contract $\mathcal{M}^{H}$ once with the charge vector. This implies the independence of the vector field-strengths at the horizon from $\varphi_{\text {flat }}$. 
On general grounds, using the properties of $\mathcal{M}(\varphi)$, one can show that if we act on the solution by means of an element $g$ of $G$, which maps $\varphi$ into $\varphi^{\prime}$ and $\mathcal{Q}$ into $\mathcal{Q}^{\prime}$, the matrix $\mathcal{M}(\varphi)$ at the horizon transforms as follows: ${ }^{1}$

$$
\mathcal{M}^{H}\left(\mathcal{Q}^{\prime}, \varphi_{\text {flat }}^{\prime}\right)=g^{-T} \mathcal{M}^{H}\left(\mathcal{Q}, \varphi_{\text {flat }}\right) g^{-1},
$$

where, with an abuse of notation, we have denoted by $g$ also the symplectic $2 n \times 2 n$ matrix representing the corresponding $G$-element on contravariant vectors of $\mathbf{R}_{\mathcal{Q}}$.

In the absence of flat directions, the above equation suggests that $\mathcal{M}^{H}(\mathcal{Q})$ should be described in terms a symmetric, symplectic, negative-definite matrix defined on the $G$-orbit of $\mathcal{Q}$, and thus constructed out of $\mathcal{Q}$ and of $G$-invariant tensors. Restricting our analysis to symmetric models with group $G$ simple of "type $\mathrm{E}_{7}$ " [18] (with the exclusion of the degenerate cases, see footnote 7 below), for charge vectors $\mathcal{Q}$ with $I_{4}(\mathcal{Q})>0$ we could construct such a matrix $M(\mathcal{Q})$ using a simple Ansatz, which involves only $\mathcal{Q}$ and $G$-invariant tensors, and imposing the following properties of $\mathcal{M}^{H}$ :

$$
\begin{aligned}
M \mathbb{C} M & =\mathbb{C} \\
M \mathcal{Q} & =-\frac{\epsilon}{2 \sqrt{\left|I_{4}\right|}} \frac{\partial I_{4}}{\partial \mathcal{Q}},
\end{aligned}
$$

where $I_{4}=: \epsilon\left|I_{4}\right|$, and $\mathbb{C}$ is the symplectic invariant $2 n \times 2 n$ antisymmetric matrix. ${ }^{2}$ Starting from the same general Ansatz we actually find two solutions to the above equations, denoted by $M_{+}(\mathcal{Q})$ and $M_{-}(\mathcal{Q})$. For charges with $I_{4}(\mathcal{Q})>0$ and no flat directions, we give arguments in favor of the identification of one of these matrices $\left(M_{+}\right)$with $\mathcal{M}^{H}(\mathcal{Q})$. The other solution $\left(M_{-}\right)$, on the other hand, is never negative definite and has the general form:

$$
M_{-, M N}=-\frac{\partial^{2} \sqrt{\left|I_{4}(\mathcal{Q})\right|}}{\partial \mathcal{Q}^{M} \partial \mathcal{Q}^{N}}
$$

This Hessian has been considered in the literature, see [19, 20], though in different contexts.

As far as regular BPS solutions in $\mathcal{N}=2$ supergravities are concerned, the two matrices $M_{ \pm}$enjoy an interesting interpretation as the value at the horizon of two characteristic symplectic, symmetric matrices of the theories: the matrix $\mathcal{M}$ which is constructed out of the real and imaginary parts of the period matrix $\mathcal{N}_{\Lambda \Sigma}(\varphi)$ (defining the generalized theta-term and the kinetic term for the vector fields, respectively), and a matrix $\mathcal{M}^{(F)}$, constructed just as $\mathcal{M}$, but in terms of the real and imaginary parts of a different complex matrix, namely the Hessian $\mathcal{F}_{\Lambda \Sigma}$ of the holomorphic prepotential of the special Kähler manifold. In terms of the covariantly holomorphic section $V=\left(V^{M}\right)$ of the special Kähler

\footnotetext{
${ }^{1}$ Here and in the following we use the short-hand notation $g^{-T}:=\left(g^{-1}\right)^{T}$.

${ }^{2}$ Note that the second of (1.5) [15] implies

$$
\mathcal{Q}^{T} M \mathcal{Q}=-2 \sqrt{\left|I_{4}(\mathcal{Q})\right|} ;
$$

however, it can be checked that this yields the same condition (namely, (B.1) further below) on the real coefficients $A, B$ and $C$ of the Ansatz (3.9)-(3.10).
} 
manifold describing the vector multiplet scalars $z^{i}$, and of its covariant derivatives $U_{i}=$ $D_{i} V=\left(U_{i}^{M}\right)$ (we use the notations of [21]), the two matrices have the following expressions:

$$
\begin{aligned}
\mathcal{M}(z, \bar{z}) & =\mathbb{C}\left(V \bar{V}^{T}+\bar{V} V^{T}+U_{i} g^{i \bar{\jmath}} \bar{U}_{\bar{\jmath}}^{T}+\bar{U}_{\bar{\jmath}} g^{\bar{\jmath} i} U_{i}^{T}\right) \mathbb{C}, \\
\mathcal{M}^{(F)}(z, \bar{z}) & =\mathbb{C}\left(V \bar{V}^{T}+\bar{V} V^{T}-U_{i} g^{i \bar{\jmath}} \bar{U}_{\bar{\jmath}}^{T}-\bar{U}_{\bar{\jmath}} g^{\bar{\jmath} i} U_{i}^{T}\right) \mathbb{C} .
\end{aligned}
$$

The former was given in [5] and [22], and it is the real part of the identity (1.16) of [15]. On the other hand, the latter expression follows from (1.13) of [16]; furthermore, $\mathcal{Q}^{T} \mathcal{M}^{(F)}(z, \bar{z}) \mathcal{Q}$ agrees with eq. (57) of [23]. In $\mathcal{N} \geqslant 2$-extended supergravities, for charge orbits characterized by $I_{4}(\mathcal{Q})<0$, the two matrices $M_{ \pm}$, though still satisfying the second of (1.5), are anti-symplectic, namely for them the following property holds:

$$
M_{ \pm} \mathbb{C} M_{ \pm}=-\mathbb{C}
$$

The matrix $M_{+}$, in particular, for all regular charge-orbits, as opposed to $M_{-}$, has the notable property of being an automorphism of the U-duality algebra $\mathfrak{g}$, that is $\mathfrak{g}$, in the representation $\mathbf{R}_{\mathcal{Q}}$, is invariant under the adjoint action of $M_{+}$(if $I_{4}<0$, being $M_{+}$ anti-symplectic, will be characterized as an outer automorphism). On the other hand $M_{-}$ is still, in all regular orbits, identified with the Hessian (1.7). Moreover both $M_{ \pm}$are invariant, up to a sign, under Freudenthal duality at the horizon.

For a generic regular charge-orbit we will find the following relation between $\mathcal{M}^{H}$ and the automorphism $M_{+}$:

$$
\mathcal{M}^{H}=M_{+} \mathcal{A}
$$

where $\mathcal{A}$ is an involutive automorphism of $G$ in the stabilizer of $\mathcal{Q}$, depending in general on $\mathcal{Q}$ and $\varphi_{\text {flat }}$. For $I_{4}<0$, both $M_{+}$and $\mathcal{A}$ are anti-symplectic outer-automorphisms of $G$, while for $I_{4}>0, \mathcal{A} \in G$ and, in the absence of flat directions, it is the identity matrix.

Besides the interpretation in terms of $\mathcal{M}$ at the horizon, which holds only for $M_{+}$in specific orbits, the solution $M_{-}$is the symplectic metric on the $G$-orbit of $\mathcal{Q}$ [20] and thus it has a mathematical relevance per se.

The plan of the paper is the following.

In section 2, we recall some basic facts about extremal black hole solutions in extended supergravities, as well as their properties under the global symmetry of the models. This includes a review of the Freudenthal duality, and sets the stage for the discussion of our results.

In section 3, which focuses on the cases without flat directions, we construct, out of a general Ansatz involving suitable contractions of the $K$-tensor and of the symplectic metric $\mathbb{C}_{M N}$ with a number of charge vectors $\mathcal{Q}$, a symmetric matrix $M$ satisfying conditions (1.5). As anticipated above, restricting our analysis to simple "non-degenerate type $\mathrm{E}_{7}$ " $U$-duality groups, treated in subsection 3.1, we actually find, for $I_{4}(\mathcal{Q})>0$, two solutions: $M_{+}$and $M_{-}$. The former is identified with $\mathcal{M}^{H}$, while the properties of the latter are studied at the end of the same section. The definition of the matrices $M_{ \pm}$is then generalized to the $I_{4}<0$ orbit, in section 3.2; here general properties of $M_{ \pm}$, in any regular charge-orbit $I_{4} \neq 0$, are discussed. 
In section 3.3 we consider $\mathcal{N}=2$ theories, where we show that $M_{-}$, in the BPS-orbit, is identified with the matrix $\mathcal{M}^{(F)}$.

A general analysis, which includes the case of regular solutions with flat directions, is finally given in section 4 , where we also summarize the previous results.

In appendix A, we recall the main properties of the independent lowest-order invariant tensors, namely $\mathbb{C}_{M N}$ (symplectic metric) and $K_{M N P Q}$ ( $K$-tensor), in the symplectic black hole charge representation $\mathbf{R}_{\mathcal{Q}}$ of the $U$-duality groups of symmetric four-dimensional Maxwell-Einstein (super)gravity theories (to which we restrict our present investigation). Appendices B and C contain details of the derivation of some results of section 3, while appendix D, containing a discussion of anti-symplectic outer-automorphisms of the U-duality algebra, concludes the paper.

\section{Symmetry properties of extremal black holes in extended supergravi- ties}

One of the basic ingredients of the symplectic formulation of electric-magnetic duality in $\mathcal{N} \geqslant 2$-extended supergravity theories in four dimensions, whose bosonic Lagrangian reads (in the absence of gauging)

$$
\mathcal{L}=-\frac{R}{2}+\frac{1}{2} g_{i j}(\varphi) \partial_{\mu} \varphi^{i} \partial^{\mu} \varphi^{j}+\frac{1}{4} I_{\Lambda \Sigma}(\varphi) F_{\mu \nu}^{\Lambda} F^{\Sigma \mid \mu \nu}+\frac{1}{8 \sqrt{-G}} R_{\Lambda \Sigma}(\varphi) \epsilon^{\mu \nu \rho \sigma} F_{\mu \nu}^{\Lambda} F_{\rho \sigma}^{\Sigma}
$$

is the $2 n \times 2 n$ real, negative definite, symmetric matrix $\mathcal{M}[24]$ :

$$
\mathcal{M}=\left(\begin{array}{cc}
\mathbb{I} & -R \\
0 & \mathbb{I}
\end{array}\right)\left(\begin{array}{cc}
I & 0 \\
0 & I^{-1}
\end{array}\right)\left(\begin{array}{cc}
\mathbb{I} & 0 \\
-R & \mathbb{I}
\end{array}\right)=\left(\begin{array}{cc}
I+R I^{-1} R & -R I^{-1} \\
-I^{-1} R & I^{-1}
\end{array}\right),
$$

where $n$ denotes the number of Abelian vector fields, and $\mathbb{I}$ denotes the $(n)$-dimensional identity matrix. $I_{\Lambda \Sigma}$ is the kinetic vector matrix, and $R_{\Lambda \Sigma}$ enters the topological theta term in (2.1); they are usually regarded as the imaginary resp. real part of a complex kinetic matrix $\mathcal{N}_{\Lambda \Sigma}$, such that $(2.2)$ yields $\mathcal{M}=\mathcal{M}[R, I]=\mathcal{M}[\operatorname{Re}(\mathcal{N}), \operatorname{Im}(\mathcal{N})]$. Moreover, it is symplectic:

$$
\mathcal{M} \mathbb{C} \mathcal{M}=\mathcal{M}^{T} \mathbb{C} \mathcal{M}=\mathbb{C} .
$$

Let us recall the main properties of this matrix which will be relevant to our subsequent discussion.

We shall restrict our analysis to theories in which the scalar manifold is homogeneous symmetric of the form $G / H$. The symplectic structure of the generalized special geometry $[4,5]$ of scalar fields yields that $\mathcal{M}$ can be equivalently rewritten as

$$
\mathcal{M}=-\left(\mathbf{L} \mathbf{L}^{T}\right)^{-1}=-\mathbf{L}^{-T} \mathbf{L}^{-1}
$$

where $\mathbf{L}$ is an element of the $S p(2 n, \mathbb{R})$-valued symplectic bundle of generalized special geometry (in the symmetric case, it is a coset representative of $G / H$ in the representation $\mathbf{R}_{\mathcal{Q}}$ ). As anticipated in the introduction, the isometry group $G$ of the scalar manifold defines the on-shell global symmetry of the theory. Under the action of a generic isometry 
$g \in G$, mapping $\varphi$ into $\varphi^{\prime}(\varphi)$ (to be also denoted in the following by $(g \star \varphi)(\varphi)=\varphi^{\prime}(\varphi)$ ), $\mathcal{M}$ transforms as follows:

$$
\mathcal{M}\left(\varphi^{\prime}\right)=g^{-T} \mathcal{M}(\varphi) g^{-1},
$$

the matrix $g$ representing the action of $G$ on contravariant vectors in $\mathbf{R}_{\mathcal{Q}}$.

The matrix $\mathcal{M}$ is an essential ingredient for writing the equations in a manifestly symplectic-covariant way, thus making their invariance under $U$-duality group apparent. To show this, as far as the Maxwell equations are concerned, let us arrange the (Abelian) vector field strengths $F^{\Lambda}(\Lambda=0,1, \ldots, n-1$; in $\mathcal{N}=2$ theories, the naught index is reserved for the graviphoton) and their magnetic duals $G_{\Lambda}$ in a symplectic vector in the representation $\mathbf{R}_{\mathcal{Q}}$ of $G$ :

$$
H=\left(H^{M}\right)=\left(F^{\Lambda}, G_{\Lambda}\right)^{T} \quad\left({ }^{*} G_{\Lambda \mid \mu \nu}:=2 \frac{\delta \mathcal{L}}{\delta F^{\Lambda \mid \mu \nu}}\right),
$$

where * denotes, as usual, the Hodge-duality (which is anti-involutive in $D=4$ spacetime: $*^{2}=-I d$ ). This quantity satisfies the so called (twisted self-duality condition) $[24]^{3}$

$$
H=\mathbb{C} \mathcal{M}^{*} H,
$$

which is a symplectic-covariant relation expressing the dependence of $G_{\Lambda}$ on $F^{\Lambda},{ }^{*} F^{\Lambda}$ and the scalar fields. The Maxwell equations are then written, in terms of $H$, as follows:

$$
d H=0 .
$$

Notice that consistency of the twisted self-duality condition (2.7) with the anti-involutivity of the Hodge-operation is a direct consequence of the symplecticity (1.10) of $\mathcal{M}$ itself. Indeed eq. (2.7) can be written in the form:

$$
{ }^{*} H=-\mathcal{S}(\varphi) H ; \quad \mathcal{S}(\varphi):=\mathbb{C} \mathcal{M}(\varphi)
$$

Eq. (1.10) then implies that the matrix $\mathcal{S}(\varphi)$ is actually an anti-involution:

$$
\mathcal{S}^{2}(\varphi)=\mathbb{C} \mathcal{M}(\varphi) \mathbb{C M}(\varphi)=\mathbb{C}^{2}=-\mathbb{I},
$$

representing a scalar-dependent almost-complex structure [17] which can be defined in every generalized special geometry [5]. For $U$-duality ${ }^{4}$ groups $G$ of type $E_{7}$ [18], $\mathcal{S}(\varphi) \in$ $\operatorname{Aut}(\mathbf{F}) \equiv G$, where $\mathbf{F}$ denotes the corresponding Freudenthal triple system [17]; in these theories, $\mathcal{S}$ may be regarded as the projection onto the adjoint in the symmetric tensor product of the symplectic representation $\mathbf{R}_{\mathcal{Q}}$ of $G$, carried by $\mathbf{F}$ itself.

Following [17], one defines the "generalized scalar-dependent Freudenthal duality"

$$
\mathfrak{F}: H \longrightarrow \mathfrak{F}(H):=-\mathcal{S}(\varphi) H,
$$

\footnotetext{
${ }^{3}$ Throughout this paper we use for the symplectic invariant matrix the following form: $\mathbb{C}=\left(\begin{array}{cc}\mathbf{0}_{n} & \mathbb{I}_{n} \\ -\mathbb{I}_{n} & \mathbf{0}_{n}\end{array}\right)$.

${ }^{4}$ Here $U$-duality is referred to as the "continuous" symmetries of [25, 26]. Their discrete versions are the $U$-duality non-perturbative string theory symmetries introduced by Hull and Townsend [1].
} 
whose general features are discussed in the same paper. By the above properties of the matrix $\mathcal{S}(\varphi), \mathfrak{F}$ is anti-involutive: $\mathfrak{F}^{2}=-I d$. The compatibility of the two anti-involutive structures, defined by $\mathfrak{F}$ and the Hodge-operation *, directly follows from (2.7) and the anti-involutivity of $S(\varphi)$ [17]:

$$
H=-\mathfrak{F}\left({ }^{*} H\right)=-{ }^{*} \mathfrak{F}(H)
$$

The matrix $\mathcal{M}$ plays an important role in the study of the properties of black hole solutions to ungauged extended supergravities, in relation to the $U$-duality group of the model. In the background of a static, spherically symmetric, asymptotically flat, dyonic extremal black hole $(\tau:=-1 / r)$

$$
d s^{2}=-e^{2 U(\tau)} d t^{2}+e^{-2 U(\tau)}\left[\frac{d \tau^{2}}{\tau^{4}}+\frac{1}{\tau^{2}}\left(d \theta^{2}+\sin \theta d \psi^{2}\right)\right],
$$

one can introduce the symplectic vector $\mathcal{Q}=\left\{p^{\Lambda}, q_{\Lambda}\right\}$ of asymptotic magnetic and electric fluxes of $H$ as follows:

$$
\mathcal{Q}=\frac{1}{4 \pi} \int_{S^{2}} H \Leftrightarrow p^{\Lambda}=\frac{1}{4 \pi} \int_{S^{2}} F^{\Lambda}, q_{\Lambda}=\frac{1}{4 \pi} \int_{S^{2}} G_{\Lambda},
$$

$S^{2}$ being any sphere of radius $r$. The spherical symmetry requires the scalar fields to depend on $\tau$ (or equivalently $r$ ) only. The action of a generic global symmetry transformation $g$ in $G$ maps a black hole in this class, described by scalar fields $\varphi(\tau)=\left(\varphi^{i}(\tau)\right)$ and a charge vector $\mathcal{Q}$, into a solution of the same kind with a charge vector $\mathcal{Q}^{\prime}=g \mathcal{Q}$ and scalar fields $\varphi^{\prime}(\tau)=g \star \varphi(\tau)$ :

$$
g \in G:[\varphi(\tau), \mathcal{Q}] \longrightarrow[g \star \varphi(\tau), g \mathcal{Q}]
$$

The generalized Freudenthal duality in (2.11) induces a scalar-dependent transformation on the electric-magnetic charges

$$
\mathcal{Q} \longrightarrow \mathfrak{F}(\mathcal{Q})=\mathfrak{F}\left(\frac{1}{4 \pi} \int_{S^{2}} H\right):=\frac{1}{4 \pi} \int_{S^{2}} \mathfrak{F}(H)=-\mathcal{S}(\varphi) \mathcal{Q}
$$

The action of $\mathfrak{F}$ on $\mathcal{Q}$ represents the "non-critical", scalar-dependent generalization of the so-called Freudenthal duality [14], defined first in [15]. Condition (2.7) then implies that:

$$
\mathfrak{F}(\mathcal{Q})=\frac{1}{4 \pi} \int_{S^{2}}{ }^{*} H
$$

The Abelian 2-form field strengths $H$, in the background (2.13), can be written, using the matrix $\mathcal{M}$, in the following $\operatorname{Sp}(2 n, \mathbb{R})$-covariant form (cfr. e.g. [27-29])

$$
\begin{aligned}
H(\varphi, U, \mathcal{Q}) & =e^{2 U} \mathbb{C} \mathcal{M}(\varphi) \mathcal{Q} d t \wedge d \tau+\mathcal{Q} \sin \theta d \theta \wedge d \psi \\
& =-e^{2 U} \mathfrak{F}(\mathcal{Q}) d t \wedge d \tau+\mathcal{Q} \sin \theta d \theta \wedge d \psi,
\end{aligned}
$$

thus implying that (recall (2.7))

$$
{ }^{*} H(\varphi, U, \mathcal{Q})=\mathfrak{F}(H(\varphi, U, \mathcal{Q}))=e^{2 U} \mathcal{Q} d t \wedge d \tau+\mathfrak{F}(\mathcal{Q}) \sin \theta d \theta \wedge d \psi=H(\varphi, U, \mathfrak{F}(\mathcal{Q})),
$$


consistently with (2.12). Note that the dependence of $H$ on the scalars is completely encoded in $\mathcal{M}(\varphi)$, or, equivalently, in the "non-critical" Freudenthal duality $\mathfrak{F}(2.11)$.

$\mathcal{M}$ also defines the (positive definite) effective black hole potential (1.2), such that $\mathfrak{F}(2.11)$ can equivalently be defined as

$$
\mathfrak{F}: \mathcal{Q} \rightarrow \mathfrak{F}(\mathcal{Q}):=\mathbb{C} \frac{\partial V_{B H}}{\partial \mathcal{Q}} .
$$

The potential $V_{B H}(1.2)$ governs the radial evolution of the scalar fields $\varphi(\tau)$ as well as of the warp factor $U(\tau)$ :

$$
\frac{d^{2} U}{d \tau^{2}}=e^{2 U} V_{B H} \quad ; \quad \frac{d^{2} \varphi^{i}}{d \tau^{2}}=g^{i j} e^{2 U} \frac{\partial V_{B H}}{\partial \varphi^{j}} .
$$

By virtue of $(2.5), V_{B H}(\varphi, \mathcal{Q})$ is invariant under a $U$-duality transformation (2.14):

$$
\forall g \in G: V_{B H}(g \star \varphi, g \mathcal{Q})=V_{B H}(\varphi, \mathcal{Q}) .
$$

At the event horizon of an extremal black hole ${ }^{5}(\tau \rightarrow-\infty)$, the attractor mechanism [6-10] implies that, regardless of the initial (asymptotic) conditions, the scalar fields evolve towards values $\varphi_{H}^{i}(\mathcal{Q})$ which only depend, up to flat directions [30], on the quantized charges:

$$
\lim _{\tau \rightarrow-\infty} \varphi^{i}=\varphi_{H}^{i}(\mathcal{Q}) .
$$

The fixed point $\varphi_{H}$ corresponds to the minimum of $V_{B H}$ :

$$
\left.\frac{\partial V_{B H}}{\partial \varphi^{i}}\right|_{\varphi=\varphi_{H}}=0 .
$$

Flat directions, generically denoted by $\varphi_{\text {flat }}$, are scalar fields which $V_{B H}$ does not depend on, and thus they are not fixed by the above extremality condition (at least at Einsteinian level [30]). These directions in symmetric supergravities span a symmetric submanifold of the scalar manifold of the form [30]:

$$
\varphi_{\text {flat }} \in \frac{G_{0}}{H_{0}} \subset \frac{G}{H},
$$

where $G_{0}$ is the stabilizer in $G$ of the charge vector $\mathcal{Q}$ and $H_{0}$ its maximal compact subgroup.

Excluding, for the time being, the existence of $\varphi_{\text {flat }}$, which shall be dealt with separately, the $U$-duality invariance (2.18) of $V_{B H}$ implies that

$$
\varphi_{H}(g \mathcal{Q})=g \star \varphi_{H}(\mathcal{Q}) .
$$

In the near-horizon limit also $\mathcal{M}$, computed on the solution, will evolve towards a matrix $\mathcal{M}^{H}$, defined as

$$
\mathcal{M}^{H}:=\lim _{\tau \rightarrow-\infty} \mathcal{M}(\varphi(\tau))=\mathcal{M}\left(\varphi_{H}^{i}\right)=\mathcal{M}^{H}(\mathcal{Q}) .
$$

\footnotetext{
${ }^{5}$ Here we shall restrict to the so called "large", i.e. regular, extremal black holes, namely to solutions whose singularity is hidden inside an event horizon with finite area $A_{H}$. These solutions are characterized by the property $A_{H}=4 \pi \sqrt{\left|I_{4}\right|} \neq 0$, see eqs. (2.27) and (2.30) below, i.e. that the quartic invariant $I_{4}$, defined below, computed on their electric and magnetic charges, is non-vanishing.
} 
We now introduce a set of dual charges $\tilde{\mathcal{Q}}=\left(\tilde{\mathcal{Q}}^{M}\right)$ defined as:

$$
\tilde{\mathcal{Q}}:=\lim _{\tau \rightarrow-\infty} \mathfrak{F}(\mathcal{Q})=-\mathbb{C} \mathcal{M}^{H} \mathcal{Q},
$$

which defines a "critical" Freudenthal duality [14]:

$$
\mathfrak{F}_{H}(\mathcal{Q}):=\tilde{\mathcal{Q}}=-\mathcal{S}^{H} \mathcal{Q},
$$

where $\mathcal{S}^{H}:=\mathbb{C} \mathcal{M}^{H}$. It can be shown [15] that

$$
\tilde{\mathcal{Q}}=\frac{1}{\pi} \mathbb{C} \frac{\partial S_{B H}}{\partial \mathcal{Q}},
$$

where $S_{B H}$ denotes the Bekenstein-Hawking entropy $[12,13]$ of the extremal black hole (2.13), given by

$$
S_{B H}=\frac{A_{H}}{4}=-\frac{\pi}{2} \mathcal{M}_{M N}^{H} \mathcal{Q}^{M} \mathcal{Q}^{N} .
$$

Note that (2.27) implies that $\mathcal{M}^{H}$ is homogeneous of degree zero in the charges.

For $U$-duality groups of type $E_{7}[18], \tilde{\mathcal{Q}}$ can also be written as $[14,15]$

$$
\tilde{\mathcal{Q}}_{M}=\epsilon \frac{2}{\sqrt{\left|I_{4}\right|}} K_{M N P Q} \mathcal{Q}^{N} \mathcal{Q}^{P} \mathcal{Q}^{Q}
$$

where $\epsilon= \pm 1$, the index $M$ was lowered by means of $\mathbb{C}, \tilde{\mathcal{Q}}_{M}=\mathbb{C}_{N M} \tilde{\mathcal{Q}}^{N}$, and $K_{M N P Q}$ is the so-called $K$-tensor, which is the invariant tensor in the 4 -fold symmetric product of the representation $\mathbf{R}_{\mathcal{Q}}$, whose properties are summarized in appendix $\mathrm{A}$. In terms of it, one can express the invariant quartic homogeneous polynomial $I_{4}$ in the charges $\mathcal{Q}$ as:

$$
I_{4}:=K_{M N P Q} \mathcal{Q}^{M} \mathcal{Q}^{N} \mathcal{Q}^{P} \mathcal{Q}^{Q}=\epsilon\left|I_{4}\right|,
$$

thus implying that the Bekenstein-Hawking entropy $S_{B H}(2.27)$ can be written as

$$
S_{B H}=\pi \sqrt{\left|I_{4}\right|} .
$$

The above expression of the entropy is manifestly invariant under a "critical" (as well as "non-critical") Freudenthal duality, since the latter amounts to acting on the charge vector by means of $\mathcal{S}^{H}$ (or $\mathcal{S}$ in the "non-critical" case), which is an element of $G$.

Using eqs. (2.24) and (2.28), we find that the charge-dependent matrix $\mathcal{M}^{H}$ satisfies the following distinctive property:

$$
\mathcal{M}^{H} \mathcal{Q}=-\frac{\epsilon}{2 \sqrt{\left|I_{4}\right|}} \frac{\partial I_{4}}{\partial \mathcal{Q}}=-2 \frac{\epsilon}{\sqrt{\left|I_{4}\right|}} K_{M N P Q} \mathcal{Q}^{M} \mathcal{Q}^{N} \mathcal{Q}^{P} \mathcal{Q}^{Q}
$$

which clearly implies $\mathcal{Q}^{T} \mathcal{M}^{H} \mathcal{Q}=-2 \sqrt{\left|I_{4}\right|}=-2 S_{B H} / \pi$, i.e. eq. (2.27).

From the above discussion we conclude that, at the event horizon of the extremal black hole, the symplectic field strength vector

$$
H_{H}:=\lim _{\tau \rightarrow-\infty} H(\varphi(\tau))
$$


reads

$$
\begin{aligned}
H_{H} & =e^{2 U_{H}} \mathbb{C} \mathcal{M}^{H} \mathcal{Q} d t \wedge d \tau+\mathcal{Q} \sin \theta d \theta \wedge d \psi \\
& =-e^{2 U_{H}} \tilde{\mathcal{Q}} d t \wedge d \tau+\mathcal{Q} \sin \theta d \theta \wedge d \psi=-\mathfrak{F}_{H}\left({ }^{*} H_{H}\right),
\end{aligned}
$$

where $U_{H}$ is the leading order contribution in $\tau$ of the near-horizon limit of $U(\tau)$. From eq. (2.31) it follows that, in the presence of flat directions, although $\mathcal{M}^{H}$ in general depends on them, the fields strengths $H_{H}$ near the horizon do not.

The expression of the matrix $\mathcal{M}$ evaluated on the radial flow of the scalar fields in a black hole solution, can be rather complicated due to the highly non-linear dependence that $\mathcal{M}$ can have on the scalars $\varphi$ (in generic $d$-geometries, for instance, the expression of the real symmetric matrices $I_{\Lambda \Sigma}$ and $R_{\Lambda \Sigma}$ can be found e.g. in section 2 of [31]; see also appendix A of [32]). One would however expect, by virtue of the attractor mechanism, the near-horizon behavior of the matrix $\mathcal{M}$ to simplify considerably, since all the physical properties of the solution, in this limiting region, only depend on the quantized charges $p^{\Lambda}, q_{\Lambda}$. Characterizing this behavior is the main motivation of our investigation.

As previously pointed out, in the absence of flat directions, when all the scalars $\varphi$ 's are stabilized to a (purely) $\mathcal{Q}$-dependent value $\varphi_{H}(\mathcal{Q})(2.19)$ at the horizon, by the attractor mechanism, also the limiting value $\mathcal{M}^{H}$ of $\mathcal{M}$ is a function of $\mathcal{Q}$ only, see eq. (2.23). Consequently, the action of an element $g$ of the $U$-duality group $G$ on the solution, which maps the initial charge vector $\mathcal{Q}$ into $\mathcal{Q}^{\prime}=g \mathcal{Q}$, induces a linear transformation on $\mathcal{M}^{H}$, as a result of eqs. (2.5), (2.23) and (2.22):

$$
\mathcal{M}^{H}(g \mathcal{Q})=\mathcal{M}\left(\varphi_{H}(g \mathcal{Q})\right)=\mathcal{M}\left(g^{\star} \varphi_{H}(\mathcal{Q})\right)=g^{-T} \mathcal{M}\left(\varphi_{H}(\mathcal{Q})\right) g^{-1}=g^{-T} \mathcal{M}^{H}(\mathcal{Q}) g^{-1} .
$$

The above transformation property hints at some intrinsic group-theoretical characterization of $\mathcal{M}^{H}$ since any symmetric $\operatorname{Sp}(2 n, \mathbb{R})$-covariant matrix $M(\mathcal{Q})_{M N}$, built out of the charge vector $\mathcal{Q}$ and of $G$-invariant tensors, transforms under $G$ as $\mathcal{M}^{H}$ in (2.34). Certainly an $\operatorname{Sp}\left(2 n_{V}+2, \mathbb{R}\right)$-covariant, symmetric matrix $M(\mathcal{Q})$, only built out of $\mathcal{Q}$ and of $G$-invariant tensors in products of the representation $\mathbf{R}_{\mathcal{Q}}$, satisfies the above transformation property. These $G$-invariant tensors in products of the representation $\mathbf{R}_{\mathcal{Q}}$ include the symplectic-invariant metric $\mathbb{C}_{M N}$ and the rank-4 completely symmetric invariant $K$-tensor $K_{M N P Q}$ (cfr. appendix A). In the next sections we address the problem of expressing $\mathcal{M}^{H}$ in terms of a matrix $M(\mathcal{Q})$ of this kind, restricting ourselves to $D=4$ Maxwell-Einstein (super)gravity theories whose scalar manifold is a symmetric space $G / H$ (which correspond to characterizing $G$ as a group of type $E_{7}[18]$ ). We find a simple identification for the $I_{4}>0$ orbits in the absence of flat directions. For a generic orbit, on the other hand, we will be able to identify $\mathcal{M}^{H}$ with a charge-dependent $G$-covariant matrix, modulo the multiplication by an involutive automorphism of $G$ in the stabilizer of $\mathcal{Q}$.

\section{The $M$-matrix and $\mathcal{M}^{H}$ without flat directions}

In the present section we focus on a class of four-dimensional Maxwell-Einstein (super)gravity theories with symmetric scalar manifolds $G / H$. We look for a matrix $M(\mathcal{Q})_{M N}$ 
constructed out of $\mathcal{Q}$ and of the $G$-invariant structures $K_{M N P Q}, \mathbb{C}_{M N}$, which satisfy the distinctive properties $(2.3),(2.31)$ of $\mathcal{M}^{H}(\mathcal{Q})_{M N}$. These conditions turn out to be rather restrictive and, starting from a general Ansatz for $M(\mathcal{Q})$, we were able to find a solution only in the $I_{4}(\mathcal{Q})>0$ orbit. We were able instead, for a generic orbit of $\mathcal{Q}$, to find solutions to the equations

$$
M \mathbb{C} M=\epsilon \mathbb{C} ; \quad M \mathcal{Q}=-\frac{\epsilon}{2 \sqrt{\left|I_{4}\right|}} \frac{\partial I_{4}}{\partial \mathcal{Q}},
$$

where $I_{4}(\mathcal{Q})=\epsilon\left|I_{4}(\mathcal{Q})\right|$. Notice the difference between the first of the above equations and (2.3), in the presence of the sign $\epsilon$ of $I_{4}$ on the right hand side of the former. In fact, for $\epsilon=-1$, the first of eq.s (3.1) defines an anti-symplectic symmetric matrix instead of a symplectic one. For each regular orbit $\left(I_{4}>0, I_{4}<0\right)$, we find two distinct solutions $M_{ \pm}$ with different properties.

In the present investigation we shall only consider simple $U$-duality groups $G$ nondegenerate of type $\mathrm{E}_{7}[18,33]$ (see footnote 7 below), leaving the treatment of the other cases to future work. In the absence of flat directions and for $I_{4}>0(\epsilon=+1)$, we can identify one of the two matrices $\left(M_{+}\right)$with $\mathcal{M}_{M N}^{H}$. Thus, even if the definiton of $M_{ \pm}$is general, the identification $\mathcal{M}_{H}=M_{+}$turns out to hold only for (cfr. e.g. [30]):

1. (1/2-)BPS attractors in all $\mathcal{N}=2$ simple non-degenerate-type $\mathrm{E}_{7}$ symmetric models (we exclude from our analysis the minimal coupling ones);

2. non-BPS $Z_{H}=0$ attractors in $S T U$ model with $I_{4}>0$.

As mentioned in the Introduction, in the most general case, $\mathcal{M}^{H}$ coincides with $M_{+}$ modulo multiplication by a transformation $\mathcal{A}$ in the little group of $\mathcal{Q}$. For $I_{4}<0(\epsilon=-1)$, $\mathcal{A}$ is non-trivial also in the absence of flat directions, as in the case of the $\mathcal{N}=2 T^{3}$-model, since $M_{+}$is antisymplectic as opposed to $\mathcal{M}^{H}$.

Non-degenerate $U$-duality groups $G$ "of type $E_{7}$ " $[18,33]$ will be considered in subsections 3.1, 3.2 and 3.3. Here we first construct the solutions $M_{ \pm}$for $I_{4}>0$, discuss their geometric properties and the relation of one of them to $\mathcal{M}^{H}$. Then we move to the definition of $M_{ \pm}$in the $I_{4}<0$ case, generalizing some of their properties to all regular orbits.

\subsection{The $I_{4}>0$ case and $\mathcal{M}^{H}$}

We start by considering the orbit $I_{4}>0$ (i.e. $\epsilon=+1$ ) of $\mathcal{Q}$ and we look for a $G$-covariant symmetric matrix $M(\mathcal{Q})$, solution to the equations (1.5):

$$
\begin{aligned}
M_{M N} M_{P Q} \mathbb{C}^{N P} & =\mathbb{C}_{M Q} \\
M_{M N} \mathcal{Q}^{N} & =-\frac{1}{2 \sqrt{\left|I_{4}\right|}} \frac{\partial I_{4}}{\partial \mathcal{Q}^{M}}=-\tilde{\mathcal{Q}}_{M} .
\end{aligned}
$$

We use for $M$ the following general Ansatz $\quad(A, B, C \in \mathbb{R})$ :

$$
M_{M N}(\mathcal{Q})=\frac{A}{\left|I_{4}\right|^{3 / 2}} K_{M} K_{N}+\frac{B}{\left|I_{4}\right|^{1 / 2}} K_{M N}+\frac{C}{\left|I_{4}\right|^{1 / 2}} K_{M B_{1} B_{2}} K_{N B_{3} B_{4}} \mathbb{C}^{B_{1} B_{3}} \mathbb{C}^{B_{2} B_{4}}
$$


where:

$$
K_{M N P}:=K_{M N P Q} \mathcal{Q}^{Q}, K_{M N}:=K_{M N P Q} \mathcal{Q}^{P} \mathcal{Q}^{Q}, K_{M}:=K_{M N P Q} \mathcal{Q}^{N} \mathcal{Q}^{P} \mathcal{Q}^{Q} .
$$

The derivations below strongly rely on the properties of the $K$-tensor, for simple $G$, discussed in appendix A. By recalling $(2.28)[14,15]$, it holds that

$$
K_{M}=\frac{1}{2} \epsilon\left|I_{4}\right|^{1 / 2} \tilde{\mathcal{Q}}_{M},
$$

such that (3.4) can be rewritten as

$$
M_{M N}(\mathcal{Q})=\frac{A}{4\left|I_{4}\right|^{1 / 2}} \tilde{\mathcal{Q}}_{M} \tilde{\mathcal{Q}}_{N}+\frac{B}{\left|I_{4}\right|^{1 / 2}} K_{M N}+\frac{C}{\left|I_{4}\right|^{1 / 2}} K_{M B_{1} B_{2}} K_{N B_{3} B_{4}} \mathbb{C}^{B_{1} B_{3}} \mathbb{C}^{B_{2} B_{4}}
$$

By exploiting the identity ${ }^{6}$

$$
K_{M A_{1} A_{2}} K_{P A_{3} A_{4}} \mathbb{C}^{A_{1} A_{3}} \mathbb{C}^{A_{2} A_{4}}=-\frac{1}{6 \tau}\left[(2 \tau-1) K_{M P}+\frac{1}{12}(\tau-1) \mathbb{C}_{A_{1}(M} \mathbb{C}_{P) A_{2}} \mathcal{Q}^{A_{1}} \mathcal{Q}^{A_{2}}\right]
$$

where $\tau$ is defined in eq. (A.8), the Ansatz (3.4) (or, equivalently (3.7)) can be further simplified as

$$
\begin{aligned}
M_{M N}(\mathcal{Q}) & =\frac{A}{\left|I_{4}\right|^{3 / 2}} K_{M} K_{N}+\frac{1}{\left|I_{4}\right|^{1 / 2}}\left(B+\frac{(1-2 \tau)}{6 \tau} C\right) K_{M N}+\frac{C}{72\left|I_{4}\right|^{1 / 2}} \frac{(\tau-1)}{\tau} \mathcal{Q}_{M} \mathcal{Q}_{N} \\
& =\frac{A}{4\left|I_{4}\right|^{1 / 2}} \tilde{\mathcal{Q}}_{M} \tilde{\mathcal{Q}}_{N}+\frac{1}{\left|I_{4}\right|^{1 / 2}}\left(B+\frac{(1-2 \tau)}{6 \tau} C\right) K_{M N}+\frac{C}{72\left|I_{4}\right|^{1 / 2}} \frac{(\tau-1)}{\tau} \mathcal{Q}_{M} \mathcal{Q}_{N} .
\end{aligned}
$$

In appendix B, the real coefficients $A, B$ and $C$ in (3.4) and (3.7) are determined by exploiting the properties (3.1).

It should be remarked that a term proportional to $\mathcal{Q}_{(M} \tilde{\mathcal{Q}}_{N)}$ cannot occur in (3.7) (or, equivalently, in (3.10)), because it is not consistent with (3.3) [15].

A consistent solution to (3.2)-(3.3) within the Ansatz (3.4) can be found only for $\epsilon=+1 \Leftrightarrow I_{4}>0$, and it reads

$$
A_{ \pm}=-2 \mp 6, \quad B_{ \pm}=\frac{6(1-2 \tau \mp \tau)}{(\tau-1)}, \quad C_{ \pm}=-\frac{36 \tau(1 \pm 1)}{(\tau-1)} .
$$

The splitting into " \pm " branches generally corresponds to two independent expressions, namely $M_{+}$and $M_{-}$, in terms of suitable contractions of the $K$-tensor itself and of the symplectic metric $\mathbb{C}_{M N}$ with charge vectors $\mathcal{Q}$ 's; note that $M_{-}$lacks the term proportional to $\mathcal{Q}_{M} \mathcal{Q}_{N}$, because $C_{-}=0$. From eq.s (3.10), (3.11), see appendix B.2, we can write the two solutions in a universal form:

$$
M_{ \pm \mid M N}(\mathcal{Q})=-\frac{2 \pm 6}{\left|I_{4}\right|^{3 / 2}} K_{M} K_{N} \pm \frac{6}{\left|I_{4}\right|^{1 / 2}} K_{M N}-\frac{1 \pm 1}{2\left|I_{4}\right|^{1 / 2}} \mathcal{Q}_{M} \mathcal{Q}_{N}
$$

\footnotetext{
${ }^{6} \mathrm{As}$ discussed in [34] and in [33], this is a consequence of a general identity involving the quantity $K_{M N P A_{1}} K_{P Q R A_{2}} \mathbb{C}^{A_{1} A_{2}}$, given by (5.16) of [34].
} 
This " \pm " ambiguity can be removed when considering the relation to the negativedefinite matrix $\mathcal{M}^{H}$. Indeed $M_{-}(\mathcal{Q})$ always has (at least) a positive eigenvalue and thus can never be identified with $\mathcal{M}^{H}$. This result is illustrated in appendix $\mathrm{C}$ by a direct computation in the STU model (and its rank-2 $\left(S T^{2}\right)$ and rank-1 $\left(T^{3}\right)$ "degenerations" determine the corresponding symmetric models), and thus holds at least in all rank-3 symmetric models of which the $S T U$ one is a universal sector. This check allows one to conclude that only the "+" branch can be consistent with the properties required for the matrix $\mathcal{M}$ (at the horizon).

Using (3.12), direct computations in the STU model and its contractions (e.g. the $T^{3}$ model) suggests the following identification (recall $I_{4}>0$ )

$$
\begin{aligned}
\mathcal{M}_{M N}^{H}(\mathcal{Q})=M_{+\mid M N}(\mathcal{Q}) & =-\frac{1}{\sqrt{I_{4}}}\left(\frac{8}{I_{4}} K_{M} K_{N}-6 K_{M N}+\mathcal{Q}_{M} \mathcal{Q}_{N}\right) \\
& =-\frac{1}{\sqrt{I_{4}}}\left(2 \tilde{\mathcal{Q}}_{M} \tilde{\mathcal{Q}}_{N}-6 K_{M N}+\mathcal{Q}_{M} \mathcal{Q}_{N}\right)
\end{aligned}
$$

which, as far as the STU model is concerned, holds for both the BPS and non-BPS orbits $I_{4}>0$.

Let us now show that, once proven for the STU model (and its "degenerations" $\mathrm{ST}^{2}$ and $\mathrm{T}^{3}$ models), the above identification holds for the BPS solutions to any symmetric $\mathcal{N}=2$ theory of which the STU model (or its "degenerations") is a consistent truncation. ${ }^{7}$ These comprise all the theories originating from dimensional reduction from $D=5$ and include the "magical" ones [39, 40]. The corresponding symmetric special Kähler manifold $G / H$ has the isotropy group $H$ of the form $H=\mathrm{U}(1) \times \mathcal{H}_{0}$, where $\mathcal{H}_{0}$ is the compact real form of the duality group in $D=5$ and is also isomorphic in $G$ to the stability group $G_{0}$ of a charge vector $\mathcal{Q}$ in the BPS orbit. This group, being compact, coincides with its maximal compact subgroup $H_{0}$, so that $\mathcal{H}_{0}$ and $H_{0}$ are isomorphic in $G$. With respect to $\mathcal{H}_{0}$ (or, equivalently $H_{0}$ ) the representation $\mathbf{R}_{\mathcal{Q}}$ branches as follows:

$$
\mathbf{R}_{\mathcal{Q}} \stackrel{\mathcal{H}_{0}}{\longrightarrow} \mathbf{1}+\mathbf{R}+\overline{\mathbf{1}}+\overline{\mathbf{R}}
$$

where $\mathbf{R}$ is, for the "magical" theories, an irreducible representation. We can choose a representative $\mathcal{Q}$ of the BPS orbit whose stabilizer $H_{0}$ coincides with the isotropy group $\mathcal{H}_{0}$ of the manifold. The components of the vector $\mathcal{Q}$ correspond to the singlets $\mathbf{1}+\overline{\mathbf{1}}$ in (3.14). The charges in the STU truncation comprise these two singlets and six components in the representation $\mathbf{R}+\overline{\mathbf{R}}$, defining the normal form of a generic element of $\mathbf{R}$ with respect to the action of $\mathcal{H}_{0}$. Both the two matrices $\mathcal{M}^{H}(\mathcal{Q})$ and $M_{+}(\mathcal{Q})$ commute with $\mathcal{H}_{0}$ :

$$
\forall h \in \mathcal{H}_{0}:\left\{\begin{array} { l } 
{ h \mathcal { M } ^ { H } ( \mathcal { Q } ) h ^ { T } = \mathcal { M } ^ { H } ( h \mathcal { Q } ) = \mathcal { M } ^ { H } ( \mathcal { Q } ) } \\
{ h M _ { + } ( \mathcal { Q } ) h ^ { T } = M _ { + } ( h \mathcal { Q } ) = M _ { + } ( \mathcal { Q } ) }
\end{array} \Leftrightarrow \left\{\begin{array}{l}
{\left[h, \mathcal{M}^{H}(\mathcal{Q})\right]=0} \\
{\left[h, M_{+}(\mathcal{Q})\right]=0}
\end{array}\right.\right.
$$

\footnotetext{
${ }^{7}$ This class of models have the feature that $G$ is of type $\mathrm{E}_{7}$ and does include the minimal-coupling models with special Kähler manifold $\frac{\mathrm{SU}(1, n)}{\mathrm{U}(n)}$ only as a degenerate [33] instance, which we shall not be dealing with in this paper. An other class of degenerate-type $\mathrm{E}_{7}$ models are the $\mathcal{N}=3$ supergravities, with scalar manifold $\frac{\mathrm{SU}(3, n)}{\mathrm{S}[\mathrm{U}(3) \times \mathrm{U}(n)]}$, which will be dealt with elsewhere.
} 
where we have used the properties that the symplectic duality action of $\mathcal{H}_{0}$ is represented by orthogonal matrices and that $\mathcal{H}_{0}$ is the stabilizer of $\mathcal{Q}$. If $\mathbf{R}$ is irreducible, by Schur's lemma, $\mathcal{M}^{H}(\mathcal{Q})$ and $M_{+}(\mathcal{Q})$ are both proportional to the identity on $\mathbf{R}$ and thus, since they coincide on the STU model, which comprise charges in $\mathbf{R}$, they do coincide on the whole $\mathbf{R}_{\mathcal{Q}}$.

As for the infinite series of models with special Kähler manifold $\frac{\mathrm{SL}(2, \mathbb{R})}{\mathrm{SO}(2)} \times \frac{\mathrm{SO}(2, n)}{\mathrm{SO}(2) \times \mathrm{SO}(n)}$, with $\mathcal{H}_{0}=\mathrm{SO}(2) \times \mathrm{SO}(n), \mathbf{R}$ is reducible, being $\mathbf{R}=\mathbf{1}+\mathbf{n}$. In these cases we did not derive the explicit form of the solutions to (3.2), (3.3) in terms of the covariant building blocks defined above, and we leave this task for a future investigation. Here, we limit ourselves to remark that, if we had the explicit form for the solution $M_{M N}$ which reduces to $M_{+}$once truncated to the STU model, by the same token, since the STU truncation comprises four charges in $\mathbf{n}+\overline{\mathbf{n}}$, the identification $\mathcal{M}^{H}=M$ would hold for the BPS solutions to these models, as well.

Notice that the above argument does not apply to the $\mathcal{N}>2$ models in which the BPS solutions have non-trivial flat directions since, with respect to the maximal compact subgroup $H_{0}$ of the stabilizer $G_{0}$ of $\mathcal{Q}$ in $G$, the representation $\mathbf{R}$ in (3.14) is generally reducible: $\mathbf{R}=\mathbf{R}_{1}+\mathbf{R}_{2}+\ldots$. Moreover $\mathcal{M}^{H}$ depends on both $\mathcal{Q}$ and $\varphi_{\text {flat }}$, and thus it commutes with $H_{0}$ only at $\varphi_{\text {flat }}=0$, being $H_{0}$ the stabilizer of this point. If however the charges of the STU truncation belong to the $\mathbf{1}+\overline{\mathbf{1}}+\mathbf{R}_{1}+\overline{\mathbf{R}}_{1}$, we can at least state that $\mathcal{M}^{H}$, at $\varphi_{\text {flat }}=0$, and $M_{+}$should coincide on the corresponding subspace. Consider, for instance, the $\mathcal{N}=8$ theory. In this case $G=\mathrm{E}_{7(7)}, G_{0}=\mathrm{E}_{6(2)}, H_{0}=\mathrm{SU}(2) \times \mathrm{SU}(6)$ and the representation $\mathbf{R}_{\mathcal{Q}}=\mathbf{5 6}$ branches as:

$$
56 \stackrel{H_{0}}{\longrightarrow} 1+(\mathbf{1}, 15)+(2,6)+\overline{\mathbf{1}}+\overline{(\mathbf{1}, \mathbf{1 5})}+\overline{(\mathbf{2 , 6 )}},
$$

The charges of the STU truncation are in the $\mathbf{1}+(\mathbf{1}, \mathbf{1 5})+\overline{\mathbf{1}}+\overline{(\mathbf{1 , 1 5})}$ and thus we expect $\mathcal{M}^{H}$, at $\varphi_{\text {flat }}=0$, and $M_{+}$to coincide on these representations, though not on the $(\mathbf{2}, \mathbf{6})+\overline{(\mathbf{2 , 6})} .^{8}$

There is another notable property of both $M_{+}$and $\mathcal{M}^{H}$ which is not shared by $M_{-}$: just as for $\mathcal{M}^{H}$, the adjoint action of $M_{+}$is an automorphism of $G$, namely

$$
\left(M_{+}\right)^{-1} \hat{R}_{\mathcal{Q}}[G] M_{+} \subset \hat{R}_{\mathcal{Q}}[G] \Leftrightarrow M_{+} \in \operatorname{Aut}(G)
$$

where $\hat{R}_{\mathcal{Q}}$ denotes the $2 n \times 2 n$ matrix representation of $G$ in $\mathbf{R}_{\mathcal{Q}}$. The above property was verified by computing the adjoint action of $M_{+}$on the Lie algebra $\mathfrak{g}$ of $G$, in the representation $\mathbf{R}_{\mathcal{Q}}$, and proving that it maps the algebra into itself.

Let us comment on the properties of the matrices $M_{ \pm}$under Freudenthal duality $\mathfrak{F}$ (2.11), and in particular under its "critical" / horizon version $\mathfrak{F}_{H}(2.26)$. By exploiting the properties of groups "of type $E_{7}$ " [18], one can show that

$$
\mathfrak{F}_{H}\left(K_{M N}\right)=K_{M N P Q} \tilde{\mathcal{Q}}^{P} \tilde{\mathcal{Q}}^{Q}=K_{M N}-\frac{1}{6} \tilde{\mathcal{Q}}_{M} \tilde{\mathcal{Q}}_{N}+\frac{1}{6} \mathcal{Q}_{M} \mathcal{Q}_{N},
$$

\footnotetext{
${ }^{8}$ Although, at a generic point $\varphi_{\text {flat }} \neq 0, \mathcal{M}^{H}\left(\mathcal{Q}, \varphi_{\text {flat }}\right)$ does not commute with $H_{0}$, the matrix $\mathcal{S}^{H}\left(\mathcal{Q}, \varphi_{\text {flat }}\right)=\mathbb{C} \mathcal{M}^{H}\left(\mathcal{Q}, \varphi_{\text {flat }}\right)$ commutes with the group $H_{0}^{\prime}$ isomorphic to $H_{0}$ in $G_{0}$ and stabilizer of $\varphi_{\text {flat }}$ (in the following we shall use the same symbol $H_{0}$ for the two isomorphic subgroups of $G_{0}$ ). As a consequence of this, one can state on general grounds that $\mathcal{S}^{H}\left(\mathcal{Q}, \varphi_{\text {flat }}\right)$ is proportional to the identity on the irreducible representations of $H_{0}^{\prime}$ in the decomposition of $\mathbf{R}_{\mathcal{Q}}$.
} 


\begin{tabular}{|c|c|c|c|}
\hline$J_{3}$ & $G$ & $\mathbf{R}_{\mathcal{Q}}$ & $(d, n)$ \\
\hline$J_{3}^{\mathbb{O}_{s}}$ & $E_{7(7)}$ & $\mathbf{5 6}$ & $(133,28)$ \\
\hline$J_{3}^{\mathbb{Q}}$ & $E_{7(-25)}$ & $\mathbf{5 6}$ & $(133,28)$ \\
\hline$J_{3}^{\mathbb{H}}$ & $S O^{*}(12)$ & $\mathbf{3 2}^{(\prime)}$ & $(66,16)$ \\
\hline$J_{3}^{\mathbb{C}}$ & $S U(3,3)$ & $\mathbf{2 0}$ & $(35,10)$ \\
\hline$J_{3}^{\mathbb{R}}$ & $S p(6, \mathbb{R})$ & $\mathbf{1 4}^{\prime}$ & $(21,7)$ \\
\hline$M_{1,2}(\mathbb{O})$ & $S U(1,5)$ & $\mathbf{2 0}$ & $(35,10)$ \\
\hline $\mathbb{R}$ & $S L(2, \mathbb{R})$ & $\mathbf{4}$ & $(3,2)$ \\
$T^{3}$ & {$[S L(2, \mathbb{R})]^{3}$} & $(\mathbf{2 , 2}, \mathbf{2})$ & $(9,4)$ \\
\hline $\begin{array}{c}\mathbb{R} \oplus \mathbb{R} \oplus \mathbb{R} \\
S T U\end{array}$ & \multicolumn{1}{|l}{} \\
\hline
\end{tabular}

Table 1. Four-dimensional $U$-duality groups $G$, black hole charge representation $\mathbf{R}_{\mathcal{Q}}$, and data $d:=\operatorname{dim} \mathbf{A d j}$ and $n:=\operatorname{dim} \mathbf{R}_{\mathcal{Q}} / 2$. The corresponding scalar manifolds are the symmetric cosets $\frac{G}{H}$, where $H$ is the maximal compact subgroup (with symmetric embedding) of $G$. $\mathbb{O}, \mathbb{H}, \mathbb{C}$ and $\mathbb{R}$ respectively denote the four division algebras of octonions, quaternions, complex and real numbers, and $\mathbb{O}_{s}$ is the split form of octonions. $M_{1,2}(\mathbb{O})$ is the Jordan triple system (not upliftable to $D=5$ ) generated by $2 \times 1$ Hermitian matrices over $\mathbb{O}[39,40]$. Note that the $S T U$ model [36, 37], based on $\mathbb{R} \oplus \mathbb{R} \oplus \mathbb{R}$, is reducible, but triality symmetric. All cases pertain to models with 8 supersymmetries, with exception of $M_{1,2}(\mathbb{O})$ and $J_{3}^{\mathbb{Q}_{s}}$, related to 20 and 32 supersymmetries, respectively. The $D=5$ uplift of the $T^{3}$ model based on $\mathbb{R}$ is the pure $\mathcal{N}=2, D=5$ supergravity. $J_{3}^{\mathbb{H}}$ is related to both 8 and 24 supersymmetries, because the corresponding supergravity theories share the very same bosonic sector [39-44]. All data $d$ and $n$ satisfy the relations (3.20)-(3.22).

which, in turn, implies

$$
\mathfrak{F}_{H}\left(M_{ \pm}(\mathcal{Q})\right) \equiv M_{ \pm}\left(\mathfrak{F}_{H}(\mathcal{Q})\right)=M_{ \pm}(\mathcal{Q})
$$

Thus, the identification (3.13) is consistent with the invariance of $\mathcal{M}_{M N}^{H}$ under $\mathfrak{F}_{H}$, as given eq. (1.9) of [15]:

$$
\mathfrak{F}_{H}\left(\mathcal{M}_{M N}^{H}\right):=\mathcal{M}_{M N}^{H}(\tilde{\mathcal{Q}})=\mathcal{M}_{M N}^{H}(\mathcal{Q})
$$

Furthermore, the result (3.11), as discussed in appendix B, is constrained by the consistency condition

$$
d=\frac{3 n(2 n+1)}{n+8},
$$

relating the dimension $d$ of $G$ and the dimension $2 n$ of the black hole charge irrep. $\mathbf{R}_{\mathcal{Q}}$. As observed in [34], (3.20) actually characterizes at least all the pairs $\left(G, \mathbf{R}_{\mathcal{Q}}\right)$ related to simple 
rank-3 Euclidean Jordan algebras [39, 40] (such pairs are example of simple, non-degenerate groups "of type $E_{7}$ " [33]).

The cases related to $D=4$ Maxwell-Einstein gravity theories with local supersymmetry are reported in table 1 ; within this class, the so-called $S T U$ model $[36,37]$ is an exception: the corresponding rank-3 Jordan algebra is semi-simple $(\mathbb{R} \oplus \mathbb{R} \oplus \mathbb{R})$, but however it still satisfies (3.20).

The condition (3.20) can be further elaborated, by observing that, in all the cases under consideration, it holds that

$$
n=3 q+4
$$

thus implying

$$
d=\frac{3(3 q+4)(2 q+3)}{q+4}
$$

For $J_{3}^{\mathbb{A}_{(s)}}$-related models ("magical" (super)gravities $[39,40]$ ), the parameter $q$ can be defined as

$$
q:=\operatorname{dim}_{\mathbb{R}} \mathbb{A}_{(s)}=8,4,2,1 \text { for } \mathbb{A}_{(s)}=\mathbb{O}_{(s)}, \mathbb{H}_{(s)}, \mathbb{C}_{(s)}, \mathbb{R},
$$

while $q=-2 / 3$ and $q=0$ for $T^{3}$ and $S T U$ model, respectively (and $q=2$ for $\mathcal{N}=$ 5 theory).

Interpretation of $M_{-}$. Interestingly, also

$$
\begin{aligned}
M_{-, I_{4}>0 \mid M N}(\mathcal{Q}) & =\frac{4}{\left(I_{4}\right)^{3 / 2}} K_{M} K_{N}-\frac{6}{\sqrt{I_{4}}} K_{M N}=\frac{1}{\sqrt{I_{4}}} \tilde{\mathcal{Q}}_{M} \tilde{\mathcal{Q}}_{N}-\frac{6}{\sqrt{I_{4}}} K_{M N} \\
& =-\partial_{M} \partial_{N} \sqrt{I_{4}}
\end{aligned}
$$

can be given a meaning within the stratification of $\mathbf{R}_{\mathcal{Q}}$ into $G$-orbits.

Indeed, $M_{-, I_{4}>0 \mid M N}(3.25)$ can be regarded as the metric of the non-compact pseudoRiemannian rigid special Kähler manifold [20]

$$
\mathbf{M}_{I_{4}>0}:=\mathcal{O}_{I_{4}>0} \times \mathbb{R}^{+}
$$

with real dimension $2 n ; \mathcal{O}_{I_{4}>0}$ denotes the corresponding "large" $G$-orbit defined by the $G$-invariant constraint $I_{4}>0$ on the charge representation $\mathbf{R}_{\mathcal{Q}}$ of $G$; the $\mathbb{R}^{+}$factor in (3.26) simply corresponds to the non-vanishing (strictly positive) values of $I_{4}$ itself. The signature along the $\mathbb{R}^{+}$-direction is negative, while the metric on $\mathcal{O}_{I_{4}>0}$ is that of the CartanKilling metric on the coset $G / G_{0}, G_{0}$ being the stabilizer of $\mathcal{Q}$, namely its positive and negative eigenvalues correspond to the non-compact and compact generators in the coset space, respectively.

In $\mathcal{N}=2$ (symmetric) theories, two $G$-orbits are defined by the constraint $I_{4}>0$ : the $\left(\frac{1}{2}-\right)$ BPS orbit, and the non-BPS $Z_{H}=0$ orbit [38]. Let us consider for instance the $\mathcal{N}=2$ exceptional "magical theory" $[39,40]\left(G=E_{7(-25)}, \mathbf{R}_{\mathcal{Q}}=\mathbf{5 6}\right)$, for which one can 
define the two pseudo-Riemannian 56-dimensional rigid special Kähler manifolds:

$$
\begin{aligned}
& \mathbf{M}_{I_{4}>0, B P S}:=\mathcal{O}_{I_{4}>0, B P S} \times \mathbb{R}^{+}=\frac{E_{7(-25)}}{E_{6(-78)}} \times \mathbb{R}^{+} \\
& \text {metric } M_{-\mid M N} \text { with }\left(n_{+}, n_{-}\right)=(54,2) ; \\
& \mathbf{M}_{I_{4}>0, n B P S}:=\mathcal{O}_{I_{4}>0, n B P S} \times \mathbb{R}^{+}=\frac{E_{7(-25)}}{E_{6(-14)}} \times \mathbb{R}^{+} \\
& \text {metric } M_{-\mid M N} \text { with }\left(n_{+}, n_{-}\right)=(22,34) .
\end{aligned}
$$

In general, the metric $M_{-\mid M N}$ of $\mathbf{M}_{I_{4}>0, B P S}$ always has signature $\left(n_{+}, n_{-}\right)=(2 n-2,2)$. This, indeed, is nothing but the signature of the symplectic matrix $\mathcal{M}^{(F)}$, see (3.45) or (3.48) below, which will be proven in section 3.3 to coincide, for the BPS orbit, with $M_{-}$. In the example of the STU truncation, for instance, one of the two positive eigenvalues of $M_{-}(3.24)-(3.25)$ is computed in appendix $\mathrm{C}$ for the charge configuration $\left(q_{0}, p^{1}, p^{2}, p^{3}\right)$, the other is implied by $M_{-}$being symplectic.

On the other hand, in the maximal $\mathcal{N}=8$ theory $\left(G=E_{7(7)}, \mathbf{R}_{\mathcal{Q}}=\mathbf{5 6}\right)$ there is only one $G$-orbit defined by the constraint $I_{4}>0$, namely the $\frac{1}{8}$-BPS "large" orbit, which thus allows to define the pseudo-Riemannian 56-dimensional rigid special Kähler manifold [20]:

$$
\begin{aligned}
\mathbf{M}_{I_{4}>0, \frac{1}{8}-B P S}:=\mathcal{O}_{I_{4}>0, \frac{1}{8}-B P S} & \times \mathbb{R}^{+}=\frac{E_{7(7)}}{E_{6(2)}} \times \mathbb{R}^{+} \\
& \text {metric } M_{-\mid M N} \text { with }\left(n_{+}, n_{-}\right)=(30,26) .
\end{aligned}
$$

\subsection{Generalizing the solutions $M_{ \pm}$to all $I_{4} \neq 0$ orbits}

If we extend the expressions for $M_{ \pm}$, given section 3.1 , to $I_{4}<0$ :

$$
\begin{aligned}
& M_{+, I_{4}<0 M N}=\frac{1}{\left(-I_{4}\right)^{\frac{3}{2}}}\left(-8 K_{M} K_{N}+6 I_{4} K_{M N}-I_{4} \mathcal{Q}_{M} \mathcal{Q}_{N}\right), \\
& M_{-, I_{4}<0 M N}=\frac{1}{\left(-I_{4}\right)^{\frac{3}{2}}}\left(4 K_{M} K_{N}-6 I_{4} K_{M N}\right) .
\end{aligned}
$$

we find that, in contrast to the $I_{4}>0$ case, these matrices, though still satisfying the condition (3.3), are anti-symplectic, namely satisfy the first of eq.s (3.1) with $\epsilon=-1$. Under the "critical" / horizon version $\mathfrak{F}_{H}(2.26)$ of Freudenthal duality, $M_{ \pm, I_{4}<0}$ transform as follows:

$$
\mathfrak{F}_{H}\left(M_{ \pm, I_{4}<0}\right)=-M_{ \pm, I_{4}<0} .
$$

This can be proved by using

$$
\mathfrak{F}_{H}\left(K_{M N}\right)=K_{M N P Q} \tilde{\mathcal{Q}}^{P} \tilde{\mathcal{Q}}^{Q}=\epsilon K_{M N}-\frac{1}{6} \tilde{\mathcal{Q}}_{M} \tilde{\mathcal{Q}}_{N}+\frac{\epsilon}{6} \mathcal{Q}_{M} \mathcal{Q}_{N},
$$

which generalizes (3.17) for any sign $\epsilon$ of $I_{4}$. Correspondingly the properties (3.18) and (3.31) can be summarized as follows:

$$
\mathfrak{F}_{H}\left(M_{ \pm}\right)=\epsilon M_{ \pm}
$$


As far as $M_{-}$is concerned, for $I_{4}<0$, it coincides with the Hessian of $-\sqrt{-I_{4}}$. As a consequence of this, in all regular orbits, we can write, as a general property of $M_{-}$,

$$
M_{-, I_{4}>0 \mid M N}(\mathcal{Q})=-\partial_{M} \partial_{N} \sqrt{\left|I_{4}\right|} .
$$

Thus also for $I_{4}<0, M_{-}$can be given the same interpretation as for the $I_{4}>0$ case: $M_{-, I_{4}<0}$ can be regarded as the metric of the non-compact pseudo-Riemannian rigid special Kähler manifold

$$
\mathbf{M}_{I_{4}<0}:=\mathcal{O}_{I_{4}<0} \times \mathbb{R}^{+}
$$

with real dimension $2 n ; \mathcal{O}_{I_{4}<0}$ denotes the unique "large" non-BPS $G$-orbit defined by the $G$-invariant constraint $I_{4}<0$ on the charge representation $\mathbf{R}_{\mathcal{Q}}$ of $G$; the $\mathbb{R}^{+}$factor in (3.35) simply corresponds to the non-vanishing values of $\left|I_{4}\right|$ itself. For the $\mathcal{N}=2$ exceptional "magical theory" and $\mathcal{N}=8$ supergravity, the manifold (3.35) is respectively given by

$$
\begin{aligned}
& \mathcal{N}=2: G=E_{7(-25)}, \mathbf{R}_{\mathcal{Q}}=\mathbf{5 6}: \mathbf{M}_{I_{4}<0}:=\frac{E_{7(-25)}}{E_{6(-26)}} \times \mathbb{R}^{+}, \\
& \text {metric } M_{-\mid M N} \text { with }\left(n_{+}, n_{-}\right)=(28,28) ; \\
& \mathcal{N}=8: G=E_{7(7)}, \mathbf{R}_{\mathcal{Q}}=\mathbf{5 6}: \mathbf{M}_{I_{4}<0}:=\frac{E_{7(7)}}{E_{6(6)}} \times \mathbb{R}^{+}, \\
& \text {metric } M_{-\mid M N} \text { with }\left(n_{+}, n_{-}\right)=(28,28) .
\end{aligned}
$$

Interestingly, the two manifolds share the same signature.

As opposed to $M_{-}$, the adjoint action of $M_{+}$defines, just as in the $I_{4}>0$ case, an automorphism of $G$, namely satisfies eq. (3.16). Since, however, for $I_{4}<0 M_{+}$is antisymplectic, it can not be an element of $G$, because the matrix realization $\hat{R}_{\mathcal{Q}}$ of the elements of $G$ in the representation $\mathbf{R}_{Q}$ is symplectic. In appendix $\mathrm{D}$ we argue that for "type $\mathrm{E}_{7}$ " supergravities the group $G$ has an outer automorphism implemented by an antisymplectic matrix in the representation $\mathbf{R}_{\mathcal{Q}}$. Since, for $G$ simple, non-degenerate of type $E_{7}$, Out $(G)=\operatorname{Aut}(G) / \operatorname{Inn}(G)$ has order not greater than 2 (see footnote 10 below), and its non-trivial element is implemented by an antisymplectic matrix, a symplectic automorphism can only be inner (see also footnote 12). We then conclude that, for $I_{4}>0, M_{+}$ defines an inner-automorphism, and is an element of $G$, while for $I_{4}<0 M_{+}$defines an outer-automorphism.

We can define the matrix $S_{+}:=\mathbb{C} M_{+}$, which is still in $\operatorname{Aut}(G)$, since $M_{+}$is. Moreover $S_{+} \mathcal{Q}=\mathbb{C} \mathcal{M}^{H} \mathcal{Q}=-\mathfrak{F}_{H}(\mathcal{Q})$. We can then use (3.33) and write:

$$
S_{+}^{-T} M_{-}(\mathcal{Q}) S_{+}^{-1}=M_{-}\left(\mathfrak{F}_{H}(\mathcal{Q})\right)=\epsilon M_{-}(\mathcal{Q}),
$$

from which we can easily derive the following property:

$$
M_{+} \mathbb{C} M_{-} \mathbb{C} M_{+}=-\epsilon M_{-},
$$

or, equivalently:

$$
M_{-} M_{+}^{-1}=M_{+} M_{-}^{-1} .
$$

Finally it can be easily shown from their definition in both $I_{4}>0$ and $I_{4}<0$ cases, that

$$
M_{ \pm M N} \mathcal{Q}^{N}=\mathcal{M}_{M N}^{H} \mathcal{Q}^{N}=-\partial_{M} \sqrt{\left|I_{4}\right|} .
$$




\subsection{Interpretation of $M_{ \pm}$in $\mathcal{N}=2$ theories}

In the vector multiplet sector of an $\mathcal{N}=2$ supergravity, we can define two symmetric, symplectic matrices: one is the matrix $\mathcal{M}$ constructed out of the real and imaginary parts of $\mathcal{N}_{\Lambda \Sigma}$, as in (2.2), the other is a matrix $\mathcal{M}^{(F)}$ defined by having the same matrix form as in (2.2), but in terms of the real and imaginary parts of the complex $n \times n$ matrix

$$
\mathcal{F}_{\Lambda \Sigma}(X)=\frac{\partial^{2} F}{\partial X^{\Lambda} \partial X^{\Sigma}}
$$

$F(X)$ being the holomorphic prepotential, homogeneous function of degree 2 of $X^{\Lambda}(z)$ (we use the notations of [21]). We can write then:

$$
\begin{aligned}
\mathcal{M}(z, \bar{z}) & =\mathcal{M}[\operatorname{Re} \mathcal{N}, \operatorname{Im} \mathcal{N}], \\
\mathcal{M}^{(F)}(z, \bar{z}) & =\mathcal{M}[\operatorname{Re} \mathcal{F}, \operatorname{Im} \mathcal{F}],
\end{aligned}
$$

where $\mathcal{M}[R, I]$ is the function of the matrices $R, I$ defined in (2.2). As anticipated in the introduction, can write the matrix $\mathcal{M}(z, \bar{z})$ in the manifestly symplectic-covariant form $[5,22]$

$$
\mathcal{M}(z, \bar{z})=\mathbb{C}\left(V \bar{V}^{T}+\bar{V} V^{T}+U_{i} g^{i \bar{\jmath}} \bar{U}_{\bar{\jmath}}^{T}+\bar{U}_{\bar{\jmath}} g^{\bar{\jmath} i} U_{i}^{T}\right) \mathbb{C} .
$$

Note that the right hand side is the sum of two symmetric matrices:

$$
A_{1}=\mathbb{C}\left(V \bar{V}^{T}+\bar{V} V^{T}\right) \mathbb{C} ; A_{2}=\mathbb{C}\left(U_{i} g^{i \bar{\jmath}} \bar{U}_{\bar{\jmath}}^{T}+\bar{U}_{\bar{\jmath}} g^{\bar{\jmath} i} U_{i}^{T}\right) \mathbb{C},
$$

which satisfy the condition $A_{1} \mathbb{C} A_{2}=0$, which follow from the general properties: $V^{T} \mathbb{C} U_{i}=$ $\bar{V}^{T} \mathbb{C} U_{i}=0$. Therefore, if $\mathcal{M}=A_{1}+A_{2}$ is symmetric and symplectic, also $A_{1}-A_{2}$ is. The latter is just the matrix $\mathcal{M}^{(F)}$ :

$$
\mathcal{M}^{(F)}(z, \bar{z})=\mathbb{C}\left(V \bar{V}^{T}+\bar{V} V^{T}-U_{i} g^{i \bar{\jmath}} \bar{U}_{\bar{\jmath}}^{T}-\bar{U}_{\bar{\jmath}} g^{\bar{\jmath} i} U_{i}^{T}\right) \mathbb{C}
$$

The relation between the two matrices being then: ${ }^{9}$

$$
\mathcal{M}(z, \bar{z})=-\mathcal{M}^{(F)}(z, \bar{z})+2 \mathbb{C}\left(V \bar{V}^{T}+\bar{V} V^{T}\right) \mathbb{C},
$$

which is consistent with the relation between the lower diagonal blocks of the two matrices given e.g. in [23]:

$$
\operatorname{Im} \mathcal{N}^{-1 \Lambda \Sigma}=-\operatorname{Im} \mathcal{F}^{-1 \Lambda \Sigma}-4 L^{(\Lambda} \bar{L}^{\Sigma)}
$$

In $\mathcal{N}=2$ theories, we can express the matrix $\mathcal{M}^{(F)}$ in a form similar to eq. (2.4) for $\mathcal{M}$, namely:

$$
\mathcal{M}^{(F)}=-\mathbf{L}^{-T} \eta \mathbf{L}^{-1},
$$

where $\mathbf{L}$ is an $\operatorname{Sp}(2 n, \mathbb{R})$-matrix of the form:

$$
\mathbf{L}=\sqrt{2}\left(\operatorname{Re}(V), \operatorname{Re}\left(U_{I}\right),-\operatorname{Im}(V), \operatorname{Im}\left(U_{I}\right)\right) ;
$$

\footnotetext{
${ }^{9}$ This relation is also given in (1.13) of [16], in terms of the so-called Hesse potential (defined in (1.10) therein).
} 
moreover, $U_{I}=E_{I}{ }^{i} U_{i}, E_{I}{ }^{i}$ being the complex Vielbein matrix of the special Kähler manifold, and $\eta$ is the diagonal matrix:

$$
\eta=\operatorname{diag}\left(1,-\mathbb{I}_{n-1}, 1,-\mathbb{I}_{n-1}\right),
$$

where $\mathbb{I}_{n-1}$ denotes the $(n-1) \times(n-1)$ identity matrix.

Let us now evaluate relation (3.46) at the horizon of a regular BPS black hole (thus, with $I_{4}>0$ ) and show that it yields the relation between $M_{ \pm}$, proving thus that, if $M_{+}$ coincides with the matrix $\mathcal{M}^{H}, M_{-}$coincides with $\mathcal{M}^{(F)}$ at the horizon. To this end, we use the relations [5]:

$$
\left.2 i \bar{Z} V^{M}\right|_{\text {horizon }}=\mathcal{Q}^{M}-i \mathbb{C}^{M N} \partial_{N} \sqrt{I_{4}}=\mathcal{Q}^{M}-\frac{2 i}{\sqrt{I_{4}}} \mathbb{C}^{M N} K_{N}
$$

which hold at the horizon of the solution. Using the property that, at the horizon, $|Z|_{\text {horizon }}^{2}=\sqrt{I_{4}}$, we end up with

$$
\left.4 V^{(M} \bar{V}^{N)}\right|_{\text {horizon }}=\frac{1}{\sqrt{I_{4}}} \mathcal{Q}^{M} \mathcal{Q}^{N}+\frac{4}{\sqrt{I_{4}^{3}}} \mathbb{C}^{M P} \mathbb{C}^{N Q} K_{P} K_{Q},
$$

so that

$$
\mathcal{M}^{H}=-\left.\mathcal{M}^{(F)}\right|_{\text {horizon }}-\frac{1}{\sqrt{I_{4}}} \mathcal{Q}_{M} \mathcal{Q}_{N}-\frac{4}{\sqrt{I_{4}^{3}}} K_{M} K_{N}
$$

which is the same relation holding between $M_{+}$and $M_{-}$. Indeed, from (4.6) and (4.4), it follows that

$$
\begin{aligned}
M_{+\mid M N} & =-M_{-\mid M N}-\epsilon \frac{1}{\sqrt{\left|I_{4}\right|}} \mathcal{Q}_{M} \mathcal{Q}_{N}-\frac{1}{\sqrt{\left|I_{4}\right|}} \tilde{\mathcal{Q}}_{M} \tilde{\mathcal{Q}}_{N} \\
& =-M_{-\mid M N}-\epsilon \frac{1}{\sqrt{\left|I_{4}\right|}} \mathcal{Q}_{M} \mathcal{Q}_{N}-\frac{4}{\left|I_{4}\right|^{3 / 2}} K_{M} K_{N}
\end{aligned}
$$

which for $I_{4}>0$ reduces to the same relation (3.53).

\section{General discussion and summary of results}

We have constructed two symmetric real matrices $M_{ \pm}(\mathcal{Q})$ satisfying the conditions $(1.5)$ :

$$
\begin{aligned}
M_{ \pm}(\mathcal{Q})^{T} \mathbb{C} M_{ \pm}(\mathcal{Q}) & =\epsilon \mathbb{C} \\
\mathcal{Q}^{T} M_{ \pm}(\mathcal{Q}) \mathcal{Q} & =-2 \sqrt{\left|I_{4}\right|}
\end{aligned}
$$

where $I_{4}=: \epsilon\left|I_{4}\right|$. These matrices also satisfy relations (3.39) :

$$
M_{ \pm M N} \mathcal{Q}^{N}=\mathcal{M}_{M N}^{H} \mathcal{Q}^{N}=-\partial_{M} \sqrt{\left|I_{4}\right|}
$$

The matrix

$$
\begin{aligned}
M_{-\mid M N} & =\frac{4}{\left|I_{4}\right|^{3 / 2}} K_{M} K_{N}-\epsilon \frac{6}{\sqrt{\left|I_{4}\right|}} K_{M N} \\
& =\frac{1}{\sqrt{\left|I_{4}\right|}} \tilde{\mathcal{Q}}_{M} \tilde{\mathcal{Q}}_{N}-\epsilon \frac{6}{\sqrt{\left|I_{4}\right|}} K_{M N}=-\partial_{M} \partial_{N} \sqrt{\left|I_{4}\right|},
\end{aligned}
$$


which is never negative definite, enjoys an interpretation as symplectic metric of the corresponding $G$-orbit of $\mathcal{Q}$ (see above as well as the final part of section 3.1). Moreover it does not belong to $\operatorname{Aut}(G)$.

On the other hand, the matrix

$$
\begin{aligned}
M_{+\mid M N} & =-\frac{8}{\left|I_{4}\right|^{3 / 2}} K_{M} K_{N}+\epsilon \frac{6}{\sqrt{\left|I_{4}\right|}} K_{M N}-\epsilon \frac{1}{\sqrt{\left|I_{4}\right|}} \mathcal{Q}_{M} \mathcal{Q}_{N} \\
& =-\frac{2}{\sqrt{\left|I_{4}\right|}} \tilde{\mathcal{Q}}_{M} \tilde{\mathcal{Q}}_{N}+\epsilon \frac{6}{\sqrt{\left|I_{4}\right|}} K_{M N}-\epsilon \frac{1}{\sqrt{\left|I_{4}\right|}} \mathcal{Q}_{M} \mathcal{Q}_{N}
\end{aligned}
$$

belongs to $\operatorname{Aut}(G)$ (in particular, see below, $M_{+, I_{4}>0} \in \operatorname{Inn}(G)$ and $M_{+, I_{4}<0} \in$ $\operatorname{Aut}(G) / \operatorname{Inn}(G)=$ : $\operatorname{Out}(G)$; cfr. e.g. appendix D).

Both matrices under $\mathfrak{F}_{H}$ (2.26) transform as in (3.33).

For charges in a generic regular $G$-orbit (also in presence of flat directions), one can construct the matrix:

$$
\mathcal{A}\left(\mathcal{Q}, \varphi_{\text {flat }}\right):=M_{+}(\mathcal{Q})^{-1} \mathcal{M}^{H}\left(\mathcal{Q}, \varphi_{\text {flat }}\right)
$$

so that

$$
\mathcal{M}^{H}\left(\mathcal{Q}, \varphi_{\text {flat }}\right)=M_{+}(\mathcal{Q}) \mathcal{A}\left(\mathcal{Q}, \varphi_{\text {flat }}\right)
$$

Let us illustrate some properties of $\mathcal{A}$; as it follows from from eq. $(4.3), \mathcal{A}\left(\mathcal{Q}, \varphi_{\text {flat }}\right)$ is in the stabilizer of $\mathcal{Q}$ in $\operatorname{GL}(2 n, \mathbb{R})$. Moreover, since $M_{+} \in \operatorname{Aut}(G)$ and $\mathcal{M}^{H} \in G \subset \operatorname{Aut}(G)$, and both are invariant under $H_{0}$ (the stability group of $\varphi_{\text {flat }}$ ), also $\mathcal{A}$ is, and thus we can write:

$$
\mathcal{A}\left(\mathcal{Q}, \varphi_{\text {flat }}\right) \in \frac{\operatorname{Aut}(G)}{H_{0}} \cap \operatorname{Stab}_{\mathcal{Q}}[\mathrm{GL}(2 n, \mathbb{R})] .
$$

An important property of $\mathcal{A}$ is the following:

$$
\mathcal{A}^{T} M_{+}(\mathcal{Q}) \mathcal{A}=M_{+}\left(\mathcal{A}^{-1} \mathcal{Q}\right)=M_{+}(\mathcal{Q}),
$$

which follows from (4.9), but can be alternatively be proven using eq.s (4.7), (2.9), (4.1), and (3.33):

$$
\begin{aligned}
\mathcal{A}^{T} M_{+}(\mathcal{Q}) \mathcal{A} & =\mathcal{M}^{H} M_{+}(\mathcal{Q})^{-1} \mathcal{M}^{H}=-\mathbb{C S}^{H} M_{+}(\mathcal{Q})^{-1}\left(\mathcal{S}^{H}\right)^{T} \mathbb{C}=-\mathbb{C} M_{+}\left(\mathcal{S}^{H} \mathcal{Q}\right)^{-1} \mathbb{C} \\
& =\epsilon M_{+}\left(\mathcal{S}^{H} \mathcal{Q}\right)=\epsilon \mathfrak{F}_{H}\left(M_{+}\right)=M_{+}(\mathcal{Q}) .
\end{aligned}
$$

From this, it also follows that $\mathcal{A}$ is involutive:

$$
\mathcal{A}^{2}=\left(M_{+}\right)^{-1} \mathcal{M}^{H}\left(M_{+}\right)^{-1} \mathcal{M}^{H}=\left(M_{+}\right)^{-1} M_{+}=\mathbb{I} .
$$

Note that a property analogous to (4.11) holds for $M_{-}$:

$$
\mathcal{A}^{T} M_{-}(\mathcal{Q}) \mathcal{A}=M_{-},
$$

as it can be shown along the same lines as in (4.11) and using property (3.38).

If $I_{4}<0, M_{+}(\mathcal{Q})$ is anti-symplectic, and thus (4.7) yields that $\mathcal{A}$ is anti-symplectic as well. Therefore, as $M_{+}(\mathcal{Q})$, it defines an outer-automorphism of $G$ (see appendix D for 
a discussion on anti-symplectic outer-automorphisms of the U-duality algebra), and one can write:

$$
\begin{aligned}
M_{+}(\mathcal{Q}) & \in \operatorname{Out}(G) ; \\
\mathcal{A}_{I_{4}<0}\left(\mathcal{Q}, \varphi_{\text {flat }}\right) & \in \operatorname{Out}(G) \cap \operatorname{Stab}_{\mathcal{Q}}[\operatorname{GL}(2 n, \mathbb{R})] .
\end{aligned}
$$

In the special case of the $T^{3}$-model the $I_{4}<0$ non-BPS solution has no flat direction and thus $\mathcal{A}_{I_{4}<0}(\mathcal{Q})$ is a purely charge dependent antisymplectic matrix in the stabilizer of $\mathcal{Q}$

$$
\mathcal{M}_{\mathcal{I}_{4}<0}^{H}=M_{+}(\mathcal{Q}) \mathcal{A}_{I_{4}<0}(\mathcal{Q})
$$

Note that, at least in those cases ${ }^{10}$ in which

$$
\operatorname{Out}(G) \subset \mathbb{Z}_{2},
$$

which comprise all simple, non-degenerate type $E_{7}$ groups $G$ (including thus $\mathrm{E}_{7(7)}$ itself) [18] in $D=4$ supergravity, all non-trivial outer-automorphisms are implemented by an antisymplectic transformation.

If $I_{4}>0, M_{+}(\mathcal{Q})$ (cfr. (4.1)) is symplectic, and thus (4.7) yields that $\mathcal{A}$ is symplectic as well. Therefore, as $M_{+}(\mathcal{Q})$, it defines an inner-automorphism of $G$, and one can write (with $\mathcal{Q}$ belonging to regular $G$-orbits with $I_{4}>0 ; H_{0}=\mathcal{H}_{0}=m c s(G) / \mathrm{U}(1)$ in the BPS case, while, in the non-BPS case, it is given for instance in [38]):

$$
\begin{aligned}
M_{+}(\mathcal{Q}) & \in \operatorname{Inn}(G)=G \\
\mathcal{A}_{I_{4}>0}\left(\mathcal{Q}, \varphi_{\text {flat }}\right) & \in \frac{G}{H_{0}} \cap \operatorname{Stab}_{\mathcal{Q}}[\operatorname{Sp}(2 n, \mathbb{R})] .
\end{aligned}
$$

In the absence of flat directions $\varphi_{\text {flat }}($ such as for $\mathcal{N}=2$ regular BPS orbit), namely in those cases considered in section $3, G_{0}=H_{0}$, we have:

$$
\frac{G}{H_{0}} \cap \operatorname{Stab}_{\mathcal{Q}}[\operatorname{Sp}(2 n, \mathbb{R})]=\frac{G_{0}}{H_{0}}=\{I d\}
$$

so that property (4.19) implies

$$
\mathcal{A}_{I_{4}>0}\left(\mathcal{Q}, \varphi_{\text {flat }}\right)=I d
$$

which is consistent with the identification $\mathcal{M}^{H}=M_{+}$made in section 3 (cfr. (3.13)).

$M_{+}$as a symmetry transformation. The property of $M_{+}$of being an automorphism of $\mathfrak{g}$ implies its leaving the $K$-tensor invariant. Indeed let $\left\{t_{\alpha}^{\prime}\right\}$ denote the basis of $\mathfrak{g}$ resulting from an adjoint action of $M_{+}$on $\left\{t_{\alpha}\right\}$. Being $M_{+}$an automorphism we have:

$$
t_{\alpha}^{\prime}=M_{+}^{-1} t_{\alpha} M_{+}=M_{\alpha}^{\beta} t_{\beta} .
$$

\footnotetext{
${ }^{10} \mathrm{An}$ interesting reference in which these properties of real forms of simple Lie groups are listed is http://en.wikipedia.org/wiki/List_of_simple_Lie_groups (see also references therein). We thank G. Dall'Agata for pointing it out to us.
} 
This action clearly leaves the invariant tensor $\eta_{\alpha \beta}:=\operatorname{Tr}\left(t_{\alpha} t_{\beta}\right)$ unaltered:

$$
\eta_{\alpha \beta}:=\operatorname{Tr}\left(t_{\alpha} t_{\beta}\right)=\operatorname{Tr}\left(t_{\alpha}^{\prime} t_{\beta}^{\prime}\right)=M_{\alpha}^{\gamma} M_{\beta}^{\delta} \eta_{\gamma \delta} .
$$

As a consequence of this, using the general expression (A.10) for $K_{M N P Q}$, we conclude that the $K$-tensor expressed in terms of $t_{\alpha}$ or $t_{\alpha}^{\prime}$ coincide, i.e. that it is $M_{+}$-invariant. If $I_{4}>0, M_{+}$also leaves the symplectic form $\mathbb{C}$ invariant, and thus is an element of $\hat{R}_{\mathcal{Q}}[G]$, as previously emphasized. If, in the other hand, $I_{4}<0, M_{+}$, being anti-symplectic, does not leave $\mathbb{C}$ invariant, but can, nevertheless, be thought of as an element of the space $\hat{R}_{\mathcal{Q}}[G] \cdot \mathcal{O}$, where $\mathcal{O}$ is the involutive anti-symplectic matrix defined in appendix $\mathrm{D}$. In the former case $\left(I_{4}>0\right) M_{+}$is a charge-dependent symmetry of the theory while in the latter $\left(I_{4}<0\right)$, the presence of $\mathcal{O}$ makes $M_{+}$a symmetry only if combined with a parity or time-reversal transformation [45]. In both cases $M_{+}$, as opposed to $\mathcal{M}^{H}$ when $\varphi_{\text {flat }} \neq 0$, only depends on the charges. Although the actions of the two matrices $M_{+}$and $\mathcal{M}^{H}$ coincide on $\mathcal{Q}$ (and define the Freudenthal dual), they differ on the other fields of the theory.

In any case $M_{+}$can be characterized as a $\hat{R}_{\mathcal{Q}}[G]$-valued function for $I_{4}>0$, or $\hat{R}_{\mathcal{Q}}[G]$. $\mathcal{O}$-valued function for $I_{4}<0$, over the duality orbit of $\mathcal{Q}$.

Let us conclude with a few comments.

A special role in our discussion has been played by outer-automorphisms of the Uduality algebra which are implemented by anti-symplectic transformations. These should correspond, modulo $U$-dualities, to a discrete symmetry of ungauged extended supergravities, see appendix D, which deserves a separate discussion [45].

Finally it would be interesting to extend our analysis to "small orbits" of $\mathbf{R}_{Q}$, for which $I_{4}=0$.

\section{Acknowledgments}

MT would like to thank Prof. A. J. Di Scala, of the Department of Mathematical Sciences of Politecnico di Torino, for useful discussions. SF would like to thank M. Floratos for many interesting discussions on black hole properties related to this investigation.

The work of SF is supported by the ERC Advanced Grant no. 226455 SUPERFIELDS.

He is on leave of absence from Department of Physics and Astronomy, University of California, Los Angeles, USA.

The work of AM is supported in part by the FWO - Vlaanderen, Project No. G.0651.11, and in part by the Interuniversity Attraction Poles Programme initiated by the Belgian Science Policy (P7/37).

The work of EO is supported by Brazilian Ministry of Science and Tecnology - National Council of Scientific Development (CNPq).

The work of MT was supported by the Italian MIUR-PRIN contract 2009KHZKRX007 "Symmetries of the Universe and of the Fundamental Interactions".

\section{A The $\boldsymbol{K}$-tensor}

Let us consider a $D=4 U$-duality group $G$ of real dimension $d$, with generators $t^{\alpha}$ in the adjoint representation $(\alpha=1, \ldots, d)$. The Gaillard-Zumino [2] symplectic maximal 
embedding

$$
G \subset \operatorname{Sp}(2 n, \mathbb{R}) ; \quad \mathbf{R}_{\mathcal{Q}}=\mathbf{2 n}
$$

is provided by $(M, N=1, \ldots, 2 n)$

$$
t_{M N}^{\alpha}:=t^{\alpha}{ }_{M}^{P} \mathbb{C}_{P N}
$$

defining the Cartan-Killing metric $k_{\alpha \beta}$ of $G$ as

$$
\left(t_{\alpha \mid M}^{N} t_{\beta \mid N}^{M}\right) \equiv k_{\alpha \beta}
$$

so that $t_{\alpha \mid M}{ }^{N} t^{\alpha}{ }_{N}{ }^{M}=d$. The tensor $t^{\alpha}{ }_{M N}$ is a singlet of $G$ and, being the representation $\mathbf{R}_{\mathcal{Q}}$ symplectic, is symmetric in its symplectic indices:

$$
t^{\alpha}{ }_{M N}=t^{\alpha}{ }_{(M N)} \text {. }
$$

At least for groups $G$ "of type $E_{7}$ " [18], it is possible to construct the aforementioned rank-4 completely symmetric invariant tensor, dubbed $K$-tensor [34]:

$$
\exists ! K_{M N P Q} \equiv \mathbf{1} \in\left(\mathbf{R}_{\mathcal{Q}} \times \mathbf{R}_{\mathcal{Q}} \times \mathbf{R}_{\mathcal{Q}} \times \mathbf{R}_{\mathcal{Q}}\right)_{s},
$$

which can be generally defined as follows:

$$
\begin{aligned}
K_{M N P Q} \propto t_{(M N}^{\alpha} t_{\alpha \mid P Q)} & =\frac{1}{3}\left(t^{\alpha}{ }_{M N} t_{\alpha \mid P Q}+t^{\alpha}{ }_{M P} t_{\alpha \mid Q N}+t^{\alpha}{ }_{M Q} t_{\alpha \mid P N}\right) \\
& =\frac{1}{4 !}\left(8 t^{\alpha}{ }_{M N} t_{\alpha \mid P Q}+16 t^{\alpha}{ }_{M(P} t_{\alpha \mid Q) N}\right) .
\end{aligned}
$$

Needless to say, the prototype of groups "of type $E_{7}$ " is $E_{7}$ itself (pertaining to $\mathcal{N}=8$ and $\mathcal{N}=2$ supergravity, in its real forms $E_{7(7)}$ and $E_{7(-25)}$, respectively), with $\mathbf{R}_{\mathcal{Q}}=\mathbf{5 6}$. By following the treatment of [34], one can prove that

$$
K_{M N P Q}=\xi\left[t_{M N}^{\alpha} t_{\alpha \mid P Q}-\tau \mathbb{C}_{M(P} \mathbb{C}_{Q) N}\right],
$$

where the real constants $\xi$ and $\tau$ have been introduced; the latter can be determined by imposing the skew-tracelessness condition $\mathbb{C}^{N P} K_{M N P Q}=0$, yielding [34]

$$
\tau=\frac{d}{n(2 n+1)},
$$

whereas, by consistency with the definitions used in literature (cfr. [46], taking into account the different normalization conventions), $\xi$ is fixed as

$$
\xi=-\frac{1}{6 \tau}=-\frac{n(2 n+1)}{6 d} .
$$

Thus, the following general expression for the $K$-tensor is obtained:

$$
K_{M N P Q}=-\frac{n(2 n+1)}{6 d}\left[t_{M N}^{\alpha} t_{\alpha \mid P Q}-\frac{d}{n(2 n+1)} \mathbb{C}_{M(P} \mathbb{C}_{Q) N}\right],
$$

The formula (A.10) will be relevant to many subsequent computations (most of them reported in appendix B). By contracting the $K$-tensor with four charge vectors $\mathcal{Q}$ 's, one obtains the quartic $G$-invariant homogeneous polynomial $I_{4}$ [41] (2.29) in $\mathbf{R}_{\mathcal{Q}}$, which can therefore be rewritten as

$$
I_{4}:=K_{M N P Q} \mathcal{Q}^{M} \mathcal{Q}^{N} \mathcal{Q}^{P} \mathcal{Q}^{Q}=-\frac{1}{6 \tau} t_{M N}^{\alpha} t_{\alpha \mid P Q} \mathcal{Q}^{M} \mathcal{Q}^{N} \mathcal{Q}^{P} \mathcal{Q}^{Q} .
$$




\section{B Computing the coefficients $A, B$ and $C$}

We will here report the derivation of result (3.11), which can actually be obtained in (at least) two equivalent ways.

\section{B.1 With the invariant tensor $S_{M Q}^{\alpha \beta} \ldots$}

We start from the condition (3.3), which can be easily recast as

$$
A+\epsilon\left(B-\frac{(2 \tau-1)}{6 \tau} C\right)=-2
$$

On the other hand, the implementation of the symplectic condition (3.2) requires some further manipulations. By exploiting (3.8), one can rewrite (3.2) as

$$
\begin{aligned}
\mathbb{C}_{M Q}= & M_{M N} M_{P Q} \mathbb{C}^{N P} \\
= & \frac{1}{\left|I_{4}\right|}\left[B-C \frac{(2 \tau-1)}{6 \tau}\right]^{2} K_{N[M} K_{Q] P} \mathbb{C}^{N P} \\
& +\frac{1}{\left|I_{4}\right|} \xi\left\{\begin{array}{l}
-\frac{1}{6} A\left[B-C \frac{(2 \tau-1)}{6 \tau}\right] \\
+\frac{1}{6} C(\tau-1)\left[B-C \frac{(2 \tau-1)}{6 \tau}\right] \\
+\frac{1}{6} \epsilon A C(\tau-1)
\end{array}\right\} K_{[M} \mathbb{C}_{Q] A} \mathcal{Q}^{A},
\end{aligned}
$$

where the result (obtained by explicit computation)

$$
K_{N} K_{P Q} K_{M} \mathbb{C}^{N P}=\frac{I_{4}}{72 \tau} K_{[M} \mathbb{C}_{Q] A} \mathcal{Q}^{A}=K_{N} K_{P[Q} K_{M]} \mathbb{C}^{N P}
$$

was used. The skew-trace of (B.2) yields

$$
\begin{aligned}
2 n= & M_{M N} M_{P Q} \mathbb{C}^{N P} \mathbb{C}^{M Q} \\
= & -\frac{1}{6}(2 \tau-1)\left[B-C \frac{(2 \tau-1)}{6 \tau}\right]^{2} \\
& +\frac{1}{6}\left\{\begin{array}{l}
-\frac{1}{6} A\left[B-C \frac{(2 \tau-1)}{6 \tau}\right] \\
+\frac{1}{6} C(\tau-1)\left[B-C \frac{(2 \tau-1)}{6 \tau}\right] \\
+\epsilon A C \frac{(\tau-1)}{6 \tau}
\end{array}\right\},
\end{aligned}
$$

where the result

$$
K_{M N} K_{P Q} \mathbb{C}^{N P} \mathbb{C}^{M Q}=-\frac{(2 \tau-1)}{6 \tau} I_{4}
$$

has been taken into account.

Since the left hand side of eq. (B.2) is skew-symmetric, the only way to obtain from (B.2) a further constraint (not proportional to the skew-trace condition (B.4)) on the real coefficients $A, B$ and $C$ is to single out the terms not proportional to the symplectic metric $\mathbb{C}_{M Q}$ itself. Group theoretical arguments (cfr. e.g. appendix C of [34]) lead to the following decomposition:

$$
\begin{aligned}
K_{M N} K_{P Q} \mathbb{C}^{N P}= & \frac{1}{18 n} \frac{1}{6 \tau} I_{4} \mathbb{C}_{M Q}-\frac{2}{9 n} \frac{1}{6 \tau} K_{[M} \mathbb{C}_{Q] A} \mathcal{Q}^{A} \\
& -\frac{1}{36 \tau^{2}} t_{\alpha \mid\left(A_{1} A_{2}\right.} S_{M)(Q}^{\alpha \beta} t_{\left.\beta \mid A_{3} A_{4}\right)} \mathcal{Q}^{A_{1}} \mathcal{Q}^{A_{2}} \mathcal{Q}^{A_{3}} \mathcal{Q}^{A_{4}}
\end{aligned}
$$


where $S_{M Q}^{\alpha \beta}$ is a $G$-invariant tensor, satisfying [34]

$$
S_{M Q}^{\alpha \beta}=S_{[M Q]}^{(\alpha \beta)} ; \quad S_{M Q}^{\alpha \beta} \mathbb{C}^{M Q}=0,
$$

and the result

$$
f_{\alpha \beta \gamma} t^{\alpha}{ }_{\left(M A_{1}\right.} t^{\beta}{ }_{\left.A_{2}\right)\left(A_{3}\right.} t^{\gamma}{ }_{\left.A_{4} Q\right)} \mathcal{Q}^{A_{1}} \mathcal{Q}^{A_{2}} \mathcal{Q}^{A_{3}} \mathcal{Q}^{A_{4}}=0
$$

has been used.

Using the irreducible decomposition

$$
-\frac{1}{6 \tau} t_{\alpha \mid(M N} t_{\beta \mid P Q} S_{M) Q}^{\alpha \beta}=\mathcal{A} K_{(M N P Q} \mathbb{C}_{R) S}
$$

(where $\mathcal{A}$ is a constant to be determined), one can prove that the three terms in the right hand side of (B.6) are not independent. In fact, the following relation holds:

$$
K_{[M} \mathbb{C}_{Q] A} \mathcal{Q}^{A}=\frac{1}{4} I_{4} \mathbb{C}_{M Q}+\frac{1}{4 \mathcal{A} \tau} t_{\alpha \mid\left(A_{1} A_{2}\right.} S_{M)(Q}^{\alpha \beta} t_{\left.\beta \mid A_{3} A_{4}\right)} \mathcal{Q}^{A_{1}} \mathcal{Q}^{A_{2}} \mathcal{Q}^{A_{3}} \mathcal{Q}^{A_{4}},
$$

thus implying (B.6) to reduce to

$$
K_{M N} K_{P Q} \mathbb{C}^{N P}=-\left(1+\frac{1}{2 n \mathcal{A}}\right) \frac{1}{36 \tau^{2}} t_{\alpha \mid\left(A_{1} A_{2}\right.} S_{M)(Q}^{\alpha \beta} t_{\left.\beta \mid A_{3} A_{4}\right)} \mathcal{Q}^{A_{1}} \mathcal{Q}^{A_{2}} \mathcal{Q}^{A_{3}} \mathcal{Q}^{A_{4}} .
$$

Therefore, the finite symplecticity condition (B.2) for $\mathcal{M}^{H}$ can be rewritten as follows:

$$
\begin{aligned}
& \mathbb{C}_{M Q}= M_{M N} M_{P Q} \mathbb{C}^{N P} \\
&=-\frac{1}{24 \tau} \epsilon\left\{\begin{array}{l}
\epsilon \tau A\left[B-C \frac{(2 \tau-1)}{6 \tau}\right] \\
+C \frac{(\tau-1)}{6}\left[B-C \frac{(2 \tau-1)}{6 \tau}\right] \\
-\epsilon \frac{1}{6} A C(\tau-1)
\end{array}\right\} \mathbb{C}_{M Q} \\
&-\frac{1}{16 \mathcal{A}\left|I_{4}\right| \tau^{2}}\left\{\begin{array}{l}
\frac{2}{9}\left(\frac{1}{n}+2 \mathcal{A}\right)\left[B-C \frac{(2 \tau-1)}{6 \tau}\right]^{2} \\
+\epsilon \tau A\left[B-C \frac{(2 \tau-1)}{6 \tau}\right] \\
+C \frac{(\tau-1)}{6}\left[B-C \frac{(2 \tau-1)}{6 \tau}\right] \\
+\epsilon A C \frac{(\tau-1)}{6} \\
\times t_{\alpha \mid\left(A_{1} A_{2}\right.} S_{M)(Q}^{\alpha \beta} t_{\left.\beta \mid A_{3} A_{4}\right)} \mathcal{Q}^{A_{1}} \mathcal{Q}^{A_{2}} \mathcal{Q}^{A_{3}} \mathcal{Q}^{A_{4}} .
\end{array}\right.
\end{aligned}
$$

It is clear that $t_{\alpha \mid\left(A_{1} A_{2}\right.} S_{M)(Q}^{\alpha \beta} t_{\left.\beta \mid A_{3} A_{4}\right)}$ contains $t_{\alpha \mid A_{1} A_{2}} S_{M Q}^{\alpha \beta} t_{\beta \mid A_{3} A_{4}}$ which, due to (B.7), is orthogonal to (and thus independent of) the symplectic metric $\mathbb{C}_{M Q}$. Thus, the related coefficient has to be set to zero. This argument leads to the following independent conditions:

$$
\begin{aligned}
-\frac{\epsilon}{6 \tau}\left\{\epsilon \tau A\left[B-C \frac{(2 \tau-1)}{6 \tau}\right]+C \frac{(\tau-1)}{6}\left[B-C \frac{(2 \tau-1)}{6 \tau}\right]+\epsilon A C \frac{(\tau-1)}{6}\right\} & =4 ; \\
-\frac{1}{9} \epsilon\left[B-C \frac{(2 \tau-1)}{6 \tau}\right]^{2} & =-4 .
\end{aligned}
$$

In these relations, the real constant $\mathcal{A}$ introduced in the decomposition (B.9) has been set to

$$
\mathcal{A}=\frac{1}{2}\left(3 \tau-\frac{1}{n}\right)
$$


The result (B.15) can be achieved by noticing that, using (B.9), the following equation holds:

$$
K_{N} K_{[M} K_{Q] P} \mathbb{C}^{N P}=-\frac{1}{36 \tau}\left(\frac{1}{n}+2 \mathcal{A}\right) I_{4} K_{[M} \mathbb{C}_{Q] A} \mathcal{Q}^{A} .
$$

$K_{N} K_{[M} K_{Q] P} \mathbb{C}^{N P}$ can also be elaborated through explicit computation, and the result is given by eq. (B.3). By comparing the skew-traces of (B.16) and (B.3), (B.15) follows.

It should be stressed that eqs. (B.13) and (B.14) are consistent with the skewtracelessness condition (B.4) iff the relation (3.20) holds. This means that only two conditions out of the three ones given by eqs. (B.4), (B.13) and (B.14) are independent. The third independent condition is given by (B.1).

Thus, the solutions of the resulting system of three independent conditions on the coefficients $A, B$ and $C$ occurring in the Ansatz (3.4) read as follows:

$$
A=-2 \mp 6 \sqrt{\epsilon}, \quad B=\frac{6(1-2 \tau \mp \tau \sqrt{\epsilon})}{(\tau-1)}, \quad C=-\frac{36 \tau(1 \pm \sqrt{\epsilon})}{(\tau-1)} .
$$

Since $A, B$ and $C$ must be real, (B.17) implies that the treatment is consistent only for $I_{4}>0 \Leftrightarrow \epsilon=+1$. Then, specifying $\epsilon=+1$, (B.17) simplifies down to the final result (3.11).

We also add that the results (B.10) and (B.11) yield

$$
K_{M N} K_{P Q} \mathbb{C}^{N P}=-\frac{1}{27 \tau}\left(\frac{1}{n}+2 \mathcal{A}\right) K_{[M} \mathbb{C}_{Q] A} \mathcal{Q}^{A}+\frac{1}{18}\left(\frac{1}{n}+2 \mathcal{A}\right) \frac{1}{6 \tau} I_{4} \mathbb{C}_{M Q}
$$

Clearly, the skew-trace of the eq. (B.18) must coincide with eq. (B.5), thus implying the consistency condition (3.20).

\section{B.2 $\cdots$ and without $S_{M Q}^{\alpha \beta}$}

By inserting (B.15) into (B.18), one obtains

$$
K_{M N} K_{P Q} \mathbb{C}^{N P}=-\frac{1}{9} K_{[M} \mathbb{C}_{Q] P} \mathcal{Q}^{P}+\frac{1}{36} I_{4} \mathbb{C}_{M Q}=-\frac{1}{9} K_{[M} \mathcal{Q}_{N]}+\frac{1}{36} I_{4} \mathbb{C}_{M Q}
$$

which, by further contracting with $\mathcal{Q}^{Q}$, yields

$$
K_{M N} K_{P} \mathbb{C}^{N P}=-K_{M P} \mathbb{C}^{N P} K_{N}=\frac{1}{12} I_{4} \mathcal{Q}_{M}
$$

Results (B.19)-(B.20) actually hint for a simpler derivation of result (3.11), not involving of the use of the $G$-invariant tensor $S_{M Q}^{\alpha \beta}$ (B.7) [34] at all.

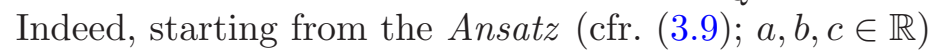

$$
M_{M N}(\mathcal{Q})=a K_{M} K_{N}+b K_{M N}+c \mathcal{Q}_{M} \mathcal{Q}_{N},
$$

and observing that ${ }^{11}$

$$
-\frac{1}{2} f_{\alpha \beta \gamma} t_{M P}^{\alpha} t_{N Q}^{\beta} t_{R S}^{\gamma} \mathcal{Q}^{P} \mathcal{Q}^{Q} \mathcal{Q}^{R} \mathcal{Q}^{S}=\tau^{2} I_{4} \mathbb{C}_{M N}+2 \tau^{2} K_{[M} \mathcal{Q}_{N]},
$$

\footnotetext{
${ }^{11}$ Note that (B.22) implies (B.8).
} 
after a little algebra eqs. (B.19)-(B.20) yield (3.11):

$$
\left\{\begin{array}{l}
a=-(2 \pm 6) /\left|I_{4}\right|^{3 / 2} \\
b= \pm 6 /\left|I_{4}\right|^{1 / 2} \\
c=-(1 \pm 1) / 2\left|I_{4}\right|^{1 / 2}
\end{array}\right.
$$

\section{Signature of $M_{-}$}

In all $\mathcal{N}$-extended, $D=4$ supergravity theories based on non-degenerate [33] $U$-duality groups $G$ "of type $E_{7}$ " [18], a generic charge vector $\mathcal{Q}$ in the $G$-repr. $\mathbf{R}_{\mathcal{Q}}$ can be $G$ transformed (through the action of a suitable element $\hat{g} \in G$ ) into a charge vector $\mathcal{Q}_{0}$ whose non-vanishing entries are only the charges $q_{0}$ and $p^{i}(i=1,2,3)$, pertaining to the $S T U$ model truncation in the special coordinates' frame (recall the absence of flat directions):

$$
\mathcal{Q} \rightarrow \mathcal{Q}_{0}=\hat{g}^{-1} \mathcal{Q} \Rightarrow M(\mathcal{Q}) \rightarrow \hat{g}^{-T} M\left(\mathcal{Q}_{0}\right) \hat{g}^{-1} .
$$

In particular, the definiteness properties of $M$ are preserved by the action of $G$.

In particular, one can consider $M_{-}(\mathcal{Q})$, given by (3.9)-(3.10) and (3.11) in the branch "-". As discussed in section $3, M_{-}$is nothing but the opposite of the Hessian of $\sqrt{I_{4}}$ (with $I_{4}>0$ ):

$$
M_{-\mid M N}=-\partial_{M} \partial_{N} \sqrt{I_{4}} .
$$

Thus, in order to study its definiteness, it suffices to analyze the signs of its diagonal elements. In the $S T U$ truncation under consideration, it can be explicitly computed that the first diagonal element is strictly positive $\left(I_{4}=q_{0} p^{1} p^{2} p^{3}>0\right)$ :

$$
M_{-\mid 00}=q_{0}^{2} \sqrt{q_{0} p^{1} p^{2} p^{3}}>0,
$$

thus implying that $M_{-\mid M N}$ is not negative definite.

On the other hand, it can be calculated that $M_{+}(\mathcal{Q})$, given by $(3.9)-(3.10)$ and $(3.11)$ in the branch "+", is diagonal, with all strictly negative elements, and thus trivially negative definite.

\section{Outer (anti-symplectic) automorphisms of $\mathfrak{g}$}

In symmetric extended $D=4$ supergravities, the U-duality algebra $\mathfrak{g}$ admits an automorphism implemented, in the representation $\mathbf{R}_{Q}$, by an anti-symplectic transformation. Consider the symplectic frame in which the elements of a suitable basis of $\mathfrak{g}_{4}$ are represented, through $\hat{R}_{\mathcal{Q}}$, either by matrices whose entries lie in the diagonal blocks or by matrices with entries only in the off-diagonal blocks. In this frame the conjugation by the anti-symplectic matrix:

$$
\mathcal{O}=\left(\begin{array}{cc}
\mathbb{I}_{n} & \mathbf{0}_{n} \\
\mathbf{0}_{n} & -\mathbb{I}_{n}
\end{array}\right),
$$

defines an automorphism:

$$
\mathcal{O}^{-1} \hat{R}_{\mathcal{Q}}[\mathfrak{g}] \mathcal{O}=\hat{R}_{\mathcal{Q}}[\mathfrak{g}],
$$


where $\hat{R}_{\mathcal{Q}}[\mathfrak{g}]$ denotes the algebra of all symplectic matrices representing $\mathfrak{g}$. For instance, in the maximal theory, such transformation switches the sign of the generators in the $\mathbf{3 5}_{c}$ (parametrized by the pseudo-scalars) and $\mathbf{3 5}_{s}$ (compact generators in $\mathfrak{s u}(8) \ominus \mathfrak{s o}(8)$ ), leaving the other generators unaltered [47].

Since all $G$ transformations in $\mathbf{R}_{\mathcal{Q}}$ are implemented by symplectic matrices, $\mathcal{O}$ is not in $G$ and defines a non-trivial outer automorphism ${ }^{12,13}$ of $\mathfrak{g}$ :

$$
\mathcal{O} \in \frac{\operatorname{Aut}(G)}{\operatorname{Inn}(G)}=\operatorname{Out}(G)
$$

We can give an alternative representation of $\mathcal{O}$, for those supergravities admitting a $D=5$ uplift, in the symplectic frame originating from the $D=5 \rightarrow D=4$ reduction. These class of models comprises all "type $\mathrm{E}_{7}$ " supergravities, excluded the "degenerate" ones, see footnote 7 . In this frame the generators $t_{\alpha}$ of $\mathfrak{g}$ have a characteristic matrix form given in [48], defined by branching the $D=4$ duality algebra with respect to $\mathrm{O}(1,1) \times$ $G_{5}, G_{5}$ being the global symmetry group of the $D=5$ parent theory. The algebra $\mathfrak{g}$ decomposes accordingly:

$$
\mathfrak{g}=\left[\mathfrak{o}(1,1) \oplus \mathfrak{g}_{5}\right]_{0} \oplus\left[\mathbf{R}_{-2}+\overline{\mathbf{R}}_{+2}\right]
$$

where the subscripts refer to $\mathrm{O}(1,1)$-gradings, $\mathbf{R}, \overline{\mathbf{R}}$ are $(n-1)$-dimensional (Abelian) spaces of nilpotent generators transforming in the representations $\mathbf{R}$ and $\overline{\mathbf{R}}$ under the adjoint action of $G_{5}$, respectively. Generators of $\mathfrak{g}$ in each of the subspaces on the righthand-side of (D.4), have the following matrix form in $\mathbf{R}_{\mathcal{Q}}$ :

$$
\begin{array}{cr}
D \in \mathfrak{o}(1,1) ; & D=\operatorname{diag}\left(-3,-\mathbb{I}_{n-1}, 3, \mathbb{I}_{n-1}\right), \\
\mathbf{E}(\lambda) \in \mathfrak{g}_{5} ; & \mathbf{E}(\lambda)=\operatorname{diag}\left(1, \mathcal{E}(\lambda), 1,-\mathcal{E}(\lambda)^{T}\right), \\
T\left(a^{I}\right) \in \overline{\mathbf{R}}_{+2} ; & T\left(a^{I}\right)=a^{I} T_{I}=\left(\begin{array}{cccc}
0 & 0 & 0 & 0 \\
a^{J} & 0 & 0 & 0 \\
0 & 0 & 0 & -a^{I} \\
0 & d_{I J} & 0 & 0
\end{array}\right), \\
\bar{T}\left(b_{I}\right) \in \mathbf{R}_{-2} ; & \bar{T}\left(b_{I}\right)=b_{I}\left(T_{I}\right)^{T},
\end{array}
$$

\footnotetext{
${ }^{12}$ Strictly speaking, to show that $\mathcal{O}$ is an outer-automorphism, one should prove that no other element of $G$ can induce the same transformation on $\mathfrak{g}$. This is immediate if $\mathbf{R}_{\mathcal{Q}}$ is irreducible since any other real matrix inducing the same transformation, must be proportional to $\mathcal{O}$, and thus non-symplectic. Inspection of supergravities in which $\mathbf{R}_{\mathcal{Q}}$ is reducible, however, leads to the same conclusion: No element of $G$ can induce the same automorphism as $\mathcal{O}$.

${ }^{13}$ The simplest example of a real Lie group admitting a symplectic representation in which an outer automorphism is implemented by an anti-symplectic transformation, is $\mathrm{SL}(2, \mathbb{R})$ : its fundamental representation 2 is symplectic and the anti-symplectic matrix $\sigma_{3}=\operatorname{diag}(+1,-1)$ (which corresponds to the limit $n=1$ in (D.5)) implements an outer-automorphism. The same holds for the spin $3 / 2$ representation 4 (with the anti-symplectic outer-automorphism given by (D.5) with $n=2)$, which also characterizes $\operatorname{SL}(2, \mathbb{R})$ as the simplest example of non-degenerate group of type $E_{7}$.
} 
where $\mathcal{E}(\lambda)$ are $(n-1) \times(n-1)$ matrices representing the generic element $\mathbf{E}(\lambda)$ of $\mathfrak{g}_{5}$. In this basis the matrix there is the following anti-symplectic automorphism $\mathcal{O}$ :

$$
\mathcal{O}=\left(\begin{array}{cccc}
1 & 0 & 0 & 0 \\
0 & -\mathbb{I}_{n-1} & 0 & 0 \\
0 & 0 & -1 & 0 \\
0 & 0 & 0 & \mathbb{I}_{n-1}
\end{array}\right),
$$

whose action on the $\mathfrak{g}$-generators is:

$$
\mathcal{O}^{-1} D \mathcal{O}=D ; \mathcal{O}^{-1} \mathbf{E}(\lambda) \mathcal{O}=\mathbf{E}(\lambda) ; \quad \mathcal{O}^{-1} T\left(a^{I}\right) \mathcal{O}=-T\left(a^{I}\right) ; \quad \mathcal{O}^{-1} \bar{T}\left(a^{I}\right) \mathcal{O}=-\bar{T}\left(a^{I}\right) .
$$

The anti-symplectic automorphism $\mathcal{O}$ is relevant for defining the $C P$-transfromation in supergravity [45].

Open Access. This article is distributed under the terms of the Creative Commons Attribution License which permits any use, distribution and reproduction in any medium, provided the original author(s) and source are credited.

\section{References}

[1] C. Hull and P. Townsend, Unity of superstring dualities, Nucl. Phys. B 438 (1995) 109 [hep-th/9410167] [INSPIRE].

[2] M.K. Gaillard and B. Zumino, Duality rotations for interacting fields, Nucl. Phys. B 193 (1981) 221 [InSPIRE].

[3] A. Strominger, Special geometry, Commun. Math. Phys. 133 (1990) 163 [InSPIRE].

[4] L. Andrianopoli, R. D'Auria and S. Ferrara, U duality and central charges in various dimensions revisited, Int. J. Mod. Phys. A 13 (1998) 431 [hep-th/9612105] [INSPIRE].

[5] S. Ferrara and R. Kallosh, On N=8 attractors, Phys. Rev. D 73 (2006) 125005 [hep-th/0603247] [INSPIRE].

[6] S. Ferrara, R. Kallosh and A. Strominger, $N=2$ extremal black holes, Phys. Rev. D 52 (1995) 5412 [hep-th/9508072] [InSPIRE].

[7] A. Strominger, Macroscopic entropy of $N=2$ extremal black holes, Phys. Lett. B 383 (1996) 39 [hep-th/9602111] [INSPIRE].

[8] S. Ferrara and R. Kallosh, Supersymmetry and attractors, Phys. Rev. D 54 (1996) 1514 [hep-th/9602136] [INSPIRE].

[9] S. Ferrara and R. Kallosh, Universality of supersymmetric attractors, Phys. Rev. D 54 (1996) 1525 [hep-th/9603090] [INSPIRE].

[10] S. Ferrara, G.W. Gibbons and R. Kallosh, Black holes and critical points in moduli space, Nucl. Phys. B 500 (1997) 75 [hep-th/9702103] [INSPIRE].

[11] B. Bertotti, Uniform electromagnetic field in the theory of general relativity, Phys. Rev. 116 (1959) 1331 [INSPIRE].

[12] S. Hawking, Gravitational radiation from colliding black holes, Phys. Rev. Lett. 26 (1971) 1344 [InSPIRE]. 
[13] J.D. Bekenstein, Black holes and entropy, Phys. Rev. D 7 (1973) 2333 [inSPIRE].

[14] L. Borsten, D. Dahanayake, M. Duff and W. Rubens, Black holes admitting a Freudenthal dual, Phys. Rev. D 80 (2009) 026003 [arXiv:0903.5517] [INSPIRE].

[15] S. Ferrara, A. Marrani and A. Yeranyan, Freudenthal duality and generalized special geometry, Phys. Lett. B 701 (2011) 640 [arXiv:1102.4857] [INSPIRE].

[16] P. Galli, P. Meessen and T. Ortín, The Freudenthal gauge symmetry of the black holes of $N=2, d=4$ supergravity, JHEP 05 (2013) 011 [arXiv:1211.7296] [INSPIRE].

[17] L. Borsten, M. Duff, S. Ferrara and A. Marrani, Freudenthal dual lagrangians, Class. Quant. Grav 30 (2013) 235003 [arXiv:1212.3254] [INSPIRE].

[18] R.B. Brown, Groups of type $E_{7}$, J. Reine Angew. Math. 236 (1969) 79.

[19] S. Ferrara and O. Macia, Observations on the Darboux coordinates for rigid special geometry, JHEP 05 (2006) 008 [hep-th/0602262] [INSPIRE].

[20] M. Graña, J. Louis, A. Sim and D. Waldram, $E_{7(7)}$ formulation of $N=2$ backgrounds, JHEP 07 (2009) 104 [arXiv:0904.2333] [INSPIRE].

[21] L. Andrianopoli et al., $N=2$ supergravity and $N=2$ super Yang-Mills theory on general scalar manifolds: symplectic covariance, gaugings and the momentum map,

J. Geom. Phys. 23 (1997) 111 [hep-th/9605032] [INSPIRE].

[22] L. Andrianopoli, R. D'Auria, E. Orazi and M. Trigiante, First order description of $D=4$ static black holes and the Hamilton-Jacobi equation, Nucl. Phys. B 833 (2010) 1 [arXiv:0905.3938] [INSPIRE].

[23] A. Ceresole, R. D'Auria and S. Ferrara, The symplectic structure of $N=2$ supergravity and its central extension, Nucl. Phys. Proc. Suppl. 46 (1996) 67 [hep-th/9509160] [InSPIRE].

[24] P. Breitenlohner, D. Maison and G.W. Gibbons, Four-dimensional black holes from Kaluza-Klein theories, Commun. Math. Phys. 120 (1988) 295 [INSPIRE].

[25] E. Cremmer and B. Julia, The $N=8$ supergravity theory. 1. The lagrangian, Phys. Lett. B 80 (1978) 48 [INSPIRE].

[26] E. Cremmer and B. Julia, The SO(8) supergravity, Nucl. Phys. B 159 (1979) 141 [inSPIRE].

[27] F. Denef, Supergravity flows and D-brane stability, JHEP 08 (2000) 050 [hep-th/0005049] [INSPIRE].

[28] L. Andrianopoli, R. D'Auria, S. Ferrara and M. Trigiante, Extremal black holes in supergravity, Lect. Notes Phys. 737 (2008) 661 [hep-th/0611345] [INSPIRE].

[29] L. Andrianopoli, R. D'Auria, S. Ferrara and M. Trigiante, Fake superpotential for large and small extremal black holes, JHEP 08 (2010) 126 [arXiv: 1002.4340] [INSPIRE].

[30] S. Ferrara and A. Marrani, On the moduli space of non-BPS attractors for $N=2$ symmetric manifolds, Phys. Lett. B 652 (2007) 111 [arXiv:0706.1667] [INSPIRE].

[31] A. Ceresole, S. Ferrara and A. Marrani, 4D/5D correspondence for the black hole potential and its critical points, Class. Quant. Grav. 24 (2007) 5651 [arXiv:0707.0964] [INSPIRE].

[32] G.L. Cardoso, J.M. Oberreuter and J. Perz, Entropy function for rotating extremal black holes in very special geometry, JHEP 05 (2007) 025 [hep-th/0701176] [INSPIRE].

[33] S. Ferrara, R. Kallosh and A. Marrani, Degeneration of groups of type $E_{7}$ and minimal coupling in supergravity, JHEP 06 (2012) 074 [arXiv: 1202.1290] [INSPIRE]. 
[34] A. Marrani, E. Orazi and F. Riccioni, Exceptional reductions, J. Phys. A 44 (2011) 155207 [arXiv: 1012.5797] [INSPIRE].

[35] L. Borsten, M. Duff, S. Ferrara, A. Marrani and W. Rubens, Small orbits, Phys. Rev. D 85 (2012) 086002 [arXiv:1108.0424] [InSPIRE].

[36] M. Duff, J.T. Liu and J. Rahmfeld, Four-dimensional string-string-string triality, Nucl. Phys. B 459 (1996) 125 [hep-th/9508094] [INSPIRE].

[37] K. Behrndt, R. Kallosh, J. Rahmfeld, M. Shmakova and W.K. Wong, STU black holes and string triality, Phys. Rev. D 54 (1996) 6293 [hep-th/9608059] [INSPIRE].

[38] S. Bellucci, S. Ferrara, M. Günaydin and A. Marrani, Charge orbits of symmetric special geometries and attractors, Int. J. Mod. Phys. A 21 (2006) 5043 [hep-th/0606209] [InSPIRE].

[39] M. Günaydin, G. Sierra and P. Townsend, Exceptional supergravity theories and the MAGIC square, Phys. Lett. B 133 (1983) 72 [InSPIRE].

[40] M. Günaydin, G. Sierra and P. Townsend, The geometry of $N=2$ Maxwell-Einstein supergravity and Jordan algebras, Nucl. Phys. B 242 (1984) 244 [INSPIRE].

[41] L. Andrianopoli, R. D'Auria and S. Ferrara, U invariants, black hole entropy and fixed scalars, Phys. Lett. B 403 (1997) 12 [hep-th/9703156] [INSPIRE].

[42] S. Ferrara, A. Gnecchi and A. Marrani, $D=4$ attractors, effective horizon radius and fake supergravity, Phys. Rev. D 78 (2008) 065003 [arXiv:0806.3196] [InSPIRE].

[43] L. Andrianopoli, R. D'Auria, S. Ferrara, P. Grassi and M. Trigiante, Exceptional $N=6$ and $N=2 A_{d} S_{4}$ Supergravity and zero-center modules, JHEP 04 (2009) 074 [arXiv:0810.1214] [INSPIRE].

[44] D. Roest and H. Samtleben, Twin supergravities, Class. Quant. Grav. 26 (2009) 155001 [arXiv: 0904.1344] [INSPIRE].

[45] P. Aschieri and M. Trigiante, Improper duality symmetries in supergravity, in preparation.

[46] L. Andrianopoli, R. D'Auria, S. Ferrara, A. Marrani and M. Trigiante, Two-centered magical charge orbits, JHEP 04 (2011) 041 [arXiv:1101.3496] [INSPIRE].

[47] G. Dall'Agata, G. Inverso and M. Trigiante, Evidence for a family of $\mathrm{SO}(8)$ gauged supergravity theories, Phys. Rev. Lett. 109 (2012) 201301 [arXiv:1209.0760] [INSPIRE].

[48] A. Ceresole, S. Ferrara, A. Gnecchi and A. Marrani, d-geometries revisited, JHEP 02 (2013) 059 [arXiv:1210.5983] [InSPIRE]. 